\title{
STABILIZED SCHEMES FOR THE HYDROSTATIC STOKES EQUATIONS*
}

\author{
F. GUILLÉN GONZÁLEZ ${ }^{\dagger}$ AND J. R. RODRÍGUEZ GALVÁN ${ }^{\ddagger}$
}

\begin{abstract}
Some new stable finite element (FE) schemes are presented for the hydrostatic Stokes system or primitive equations of the ocean. It is known that the stability of the mixed formulation approximation for primitive equations requires the well-known Ladyzhenskaya-Babuška-Brezzi condition related to the Stokes problem and an extra inf-sup condition relating the pressure and the vertical velocity [F. Guillén-González and J. R. Rodríguez-Galván, Numer. Math., 130 (2015), pp. 225-256]. The main goal of this paper is to avoid this extra condition by adding a residual stabilizing term to the vertical momentum equation. Then, the stability for Stokes-stable FE combinations is extended to the primitive equations and some error estimates are provided using Taylor-Hood $\mathcal{P}_{2}-\mathcal{P}_{1}$ or minielement $\left(\mathcal{P}_{1}+\right.$ bubble $)-\mathcal{P}_{1}$ FE approximations, showing the optimal convergence rate in the $\mathcal{P}_{2}-\mathcal{P}_{1}$ case. These results are also extended to the anisotropic (nonhydrostatic) problem. On the other hand, by adding another residual term to the continuity equation, a better approximation of the vertical derivative of pressure is obtained. In this case, stability and error estimates including this better approximation are deduced, where optimal convergence rate is deduced in the $\left(\mathcal{P}_{1}+\right.$ bubble $)-\mathcal{P}_{1}$ case. Finally, some numerical experiments are presented supporting previous results.
\end{abstract}

Key words. inf-sup condition, incompressible fluids, hydrostatic pressure, primitive equations, finite-elements, stabilized schemes

AMS subject classifications. 35Q35, 65N12, 65N30, 76D07

DOI. $10.1137 / 140998640$

1. Introduction. The purpose of this work is to present some formulations of the hydrostatic Stokes equations (or primitive equations of the ocean) for which usual Ladyzhenskaya-Babuška-Brezzi (LBB)-stable Stokes finite elements (FEs) are also stable for primitive equations. Moreover, the introduction of vertical integrated formulations (customary in most schemes from oceanography but difficult to implement) is avoided.

The interest of this work is to allow exploiting the advantages (both from the theoretical and the implementation point of view) of formulating hydrostatic Stokes equations as a mixed (Stokes-like) problem which can be approximated by standard FE and software tools.

The key in these formulations is to circumvent the main difficulty in hydrostatic and quasi-hydrostatic Stokes equations (with respect to the classical Stokes problem): the absence of viscosity in vertical momentum equation, which is reduced to the hydrostatic restriction, $\partial_{z} p=0$. In fact, for this reason, standard LBB-stable Stokes FE (like Taylor-Hood or minielement) are unfeasible and integro-differential formulations are usually introduced in hydrostatic models (see sections 1.1 and 1.2 for details). In section 2, we handle this difficulty adding a residual term which "stabilizes" the vertical velocity, obtaining also some convergence rates.

*Received by the editors December 3, 2014; accepted for publication (in revised form) April 24, 2015; published electronically August 6, 2015. The research of the authors was been partially supported by project MTM2012-32325 (MINECO, Spain).

http://www.siam.org/journals/sinum/53-4/99864.html

${ }^{\dagger}$ Departamento de Ecuaciones Diferenciales y Análisis Numérico and IMUS, Universidad de Sevilla, 41080 Sevilla, Spain (guillen@us.es).

¥Departamento de Matemáticas, Universidad de Cádiz, C.A.S.E.M. Polígono Río San Pedro S/N, 11510 Puerto Real, Cádiz, Spain (rafael.rodriguez@uca.es). The research of this author was partially supported by the research group FQM-315 (Junta de Andalucía).

1876 


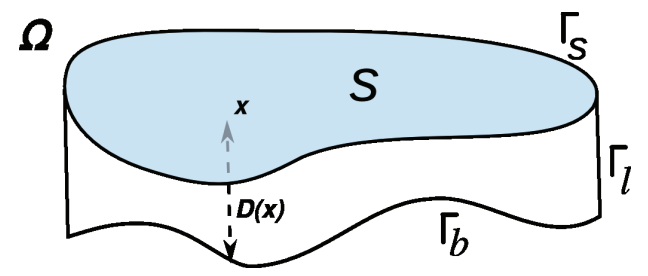

FIG. 1. Adimensionalized oceanic domain.

In addition, another residual term is considered in section 3, implying a better approximation of the vertical derivative of pressure. Numerical tests that agree with our theory are provided in section 4.

1.1. Classical hydrostatic Stokes formulations in oceanography. The equations of geophysical fluid dynamics governing the motion of the ocean and the atmosphere are derived from the conservation laws from physics. In the case of a largescale ocean (see, e.g., [10, 22, 23, 26, 27, 28]), it is considered as made up of a slightly compressible fluid modelled by conservation of momentum and mass equations, with variable density (depending on temperature and salinity) and Coriolis acceleration.

The resulting system is too complex, and, from a practical point of view, numerous simplifications are introduced, starting from Cartesian coordinates and the "small layer" hypothesis:

$$
\varepsilon=\frac{\text { vertical scale }}{\text { horizontal scale }} \quad \text { is very small, }
$$

for example, a few Kms over some thousand Kms, that is, $\varepsilon \simeq 10^{-3}, 10^{-4}$.

Second, constant density is assumed, and hence the momentum law yields to the Navier-Stokes equations for a large-scale ocean, imposed in an anisotropic domain which, after a vertical scaling, is transformed into the following isotropic or adimensional (independent on the constant $\varepsilon$ ) domain (see Figure 1):

$$
\Omega=\left\{(\mathbf{x}, z) \in \mathbb{R}^{3} / \mathbf{x}=(x, y) \in S,-D(\mathbf{x})<z<0\right\} .
$$

Here, $S \subset \mathbb{R}^{2}$ is a bounded domain in $\mathbb{R}^{2}$ (the surface domain), and $D: \bar{S} \rightarrow \mathbb{R}_{+}$is a function describing the bottom depth. The rigid lid hypothesis has been assumed (no vertical displacements of the free surface of the ocean), as usual in large-scale oceanography except in the case when fast surface waves are of interest (see, e.g., [10, section 7.5]). We decompose the boundary into three parts: the surface, $\Gamma_{s}=\bar{S} \times\{0\}$, the bottom, $\Gamma_{b}=\{(\mathbf{x},-D(\mathbf{x})) / \mathbf{x}=(x, y) \in S\}$, and the talus or lateral walls, $\Gamma_{l}=\{(\mathbf{x}, z) / \mathbf{x} \in \partial S,-D(\mathbf{x})<z<0\}$.

Also, a $\varepsilon$-dependent scaling of vertical velocity is introduced (see [3]), leading to the following anisotropic Navier-Stokes equations in the time-space domain $(0, T) \times \Omega$ :

$$
\begin{aligned}
\partial_{t} \mathbf{u}+\left(\mathbf{u} \cdot \nabla_{\mathbf{x}}\right) \mathbf{u}+v \partial_{z} \mathbf{u}-\Delta_{\nu} \mathbf{u}+\nabla_{\mathbf{x}} p & =\mathbf{f}, \\
\varepsilon^{2}\left\{\partial_{t} v+\left(\mathbf{u} \cdot \nabla_{\mathbf{x}}\right) v+v \partial_{z} v-\Delta_{\nu} v\right\}+\partial_{z} p & =g, \\
\nabla_{\mathbf{x}} \cdot \mathbf{u}+\partial_{z} v & =0,
\end{aligned}
$$

where $\nabla_{\mathbf{x}}=\left(\partial_{x}, \partial_{y}\right)^{T}, \nabla_{\mathbf{x}} \cdot \mathbf{u}=\partial_{x} u_{1}+\partial_{y} u_{2}, \Delta_{\nu}=\nu_{x} \partial_{x x}^{2}+\nu_{y} \partial_{y y}^{2}+\nu_{z} \partial_{z z}^{2}$, with $\nu=\left(\nu_{x}, \nu_{y}, \nu_{z}\right)$ being the (adimensional kinematic) viscosity. The unknowns are the three-dimensional (3D) velocity field, $(\mathbf{u}, v): \Omega \times(0, T) \rightarrow \mathbb{R}^{3}$, and the pressure, $p: \Omega \times$ 
$(0, T) \rightarrow \mathbb{R}$. The term $\mathbf{f}=\left(f_{1}, f_{2}\right)^{T}$ models a given horizontal force, while $g$ involves the force due to gravity, which can be written in potential form and incorporated to the pressure term. Hence, it can be assumed that $g=0$ in (1.2). Other phenomena like the effects due to the Coriolis acceleration are not considered in this work because they are linear terms not affecting to the results presented below. The system is endowed with initial values for the velocity field, $\left.(\mathbf{u}, v)\right|_{t=0}=\left(\mathbf{u}_{0}, v_{0}\right)$, and adequate boundary conditions, for instance,

$$
\begin{array}{r}
\left.\nu_{z} \partial_{z} \mathbf{u}\right|_{\Gamma_{s}}=\mathbf{g}_{s},\left.\quad v\right|_{\Gamma_{s}}=0, \\
\left.\mathbf{u}\right|_{\Gamma_{b} \cup \Gamma_{l}}=0,\left.\quad v\right|_{\Gamma_{b}}=0, \\
\left.\nabla_{\mathbf{x}} v \cdot \mathbf{n}_{\mathbf{x}}\right|_{\Gamma_{l}}=0,
\end{array}
$$

where $\mathbf{g}_{s}$ represents the wind stress and $\mathbf{n}_{\mathbf{x}}$ is the horizontal part of the outward normal vector. Boundary conditions are not imposed for $v$ on $\Gamma_{l}$ due to its lack of regularity when $\varepsilon \rightarrow 0$.

From a numerical point of view, as shown in [15], the strong anisotropy of NavierStokes system (1.1)-(1.3) when $\varepsilon$ is small (for instance, $\varepsilon=10^{-3}, 10^{-4}$, or smaller, which we will call "quasi-hydrostatic") affects its stability and invalidates its approximation by means of standard stable combinations of FE, such as Taylor-Hood $\mathcal{P}_{2}-\mathcal{P}_{1}$, or the minielement, $\mathcal{P}_{1, b}-\mathcal{P}_{1}$ (where, in what follows, $\mathcal{P}_{1, b}$ denotes $\left(\mathcal{P}_{1}+\right.$ bubble) FE). In fact, in [1,2] and [15], it was shown that the key is the instability of vertical velocity and it seems more adequate choosing $\mathrm{FE}$ combinations satisfying some additional restrictions (see section 1.2 for more details). In this current work, we develop strategies which avoid these restrictions.

In what follows, the less favorable case $\varepsilon=0$ will be considered. The idea is defining suitable schemes for $\varepsilon=0$, which conserve their properties uniformly when $\varepsilon>0$ and thus are also appropriate for (1.1)-(1.3). Therefore, we focus on the hydrostatic Navier-Stokes equations or primitive equations of the ocean in $(0, T) \times \Omega$,

$$
\begin{aligned}
\partial_{t} \mathbf{u}+\left(\mathbf{u} \cdot \nabla_{\mathbf{x}}\right) \mathbf{u}+v \partial_{z} \mathbf{u}-\Delta_{\nu} \mathbf{u}+\nabla_{\mathbf{x}} p & =\mathbf{f}, \\
\partial_{z} p & =0, \\
\nabla_{\mathbf{x}} \cdot \mathbf{u}+\partial_{z} v & =0,
\end{aligned}
$$

endowed with the boundary conditions (1.4) and (1.5). These equations play an important role in oceanography, where the hydrostatic approximation (1.8) is justified by means of scale analysis from the Boussinesq equations (see, e.g., $[10,26]$ ). From a mathematical point of view, the vertical velocity $v$ loose definitively its regularity in the horizontal ( $x$ and $y$ ) directions and then boundary condition (1.6) disappears. Contrary, (1.8) implies that $\partial_{z} p$ gains regularity in the vertical direction.

The obtention of (1.7)-(1.9) as a limit of (1.1)-(1.3) when $\varepsilon$ tends to zero was justified on rigorous mathematical grounds in [4] (stationary case) and [3] (evolutive case). As far as we know, all existence and regularity results (see, e.g., [23, 8, 25, $9,21,29,13,7,19])$ except $[1,2]$ are based on replacing (1.7)-(1.9) by the following equivalent integral-differential problem:

$$
\begin{array}{r}
\partial_{t} \mathbf{u}+\left(\mathbf{u} \cdot \nabla_{\mathbf{x}}\right) \mathbf{u}+v \partial_{z} \mathbf{u}-\Delta_{\nu} \mathbf{u}+\nabla_{\mathbf{x}} p_{s}=\mathbf{f} \text { in } \Omega \\
\nabla_{\mathbf{x}} \cdot\langle\mathbf{u}\rangle=0 \text { in } \Omega, \\
\mathbf{u}=0 \quad \text { on } \Gamma_{b} \cup \Gamma_{l}, \quad \nu_{z} \partial_{z} \mathbf{u}=\mathbf{g}_{s} \text { on } \Gamma_{s},
\end{array}
$$

Copyright $@$ ㅇ by SIAM. Unauthorized reproduction of this article is prohibited. 
where $p_{s}: \Omega \times(0, T) \rightarrow \mathbb{R}$ is the (artificial) surface pressure and $v$ and $\langle\mathbf{u}\rangle$ are defined by

$$
v(\mathbf{x}, z, t)=\int_{z}^{0} \nabla_{\mathbf{x}} \cdot \mathbf{u}(\mathbf{x}, s, t) d s, \quad\langle\mathbf{u}\rangle(\mathbf{x}, t)=\int_{-D(\mathbf{x})}^{0} \mathbf{u}(\mathbf{x}, z, t) d z .
$$

This reduced formulation has been also preferred for numerical schemes; see, e.g., $[16,8,9,17,18]$. In fact, it presents some advantages over (1.7)-(1.9): $v$ is decoupled, and $p$ is reduced from $\Omega$ to $S$. But, on the other hand, it requires the calculus of integrals in the $z$ variable, imposing a strong vertical structure to the FE mesh and making it difficult to use standard software tools.

From this point of view, the original hydrostatic Navier-Stokes problem (1.7)(1.9) seems more adequate, because it allows employing usual mixed velocity/pressure formulations. Its main drawback is (as commented above) the nonstability of most of the FE combinations which are standard for the Stokes problem. Now we present some results which delve into in this question and are useful in the rest of this work.

1.2. Recent results about nonintegral mixed formulations. In this section, we summarize some results, extracted from in [15] and references therein (mainly $[1,2])$, where the mixed formulation of the steady linear system related to (1.7)(1.9) is studied (without integro-differential formulations), providing the existence and uniqueness of weak solution $(\mathbf{u}, v, p) \in \mathbf{U} \times V \times P$ of the linear system

$$
\begin{aligned}
\nu(\nabla \mathbf{u}, \nabla \overline{\mathbf{u}})-\left(p, \nabla_{\mathbf{x}} \cdot \overline{\mathbf{u}}\right) & =\langle\mathbf{f}, \overline{\mathbf{u}}\rangle & & \forall \overline{\mathbf{u}} \in \mathbf{U}, \\
\left(p, \partial_{z} \bar{v}\right) & =0 & & \forall \bar{v} \in V, \\
(\nabla \cdot(\mathbf{u}, v), \bar{p}) & =0 & & \forall \bar{p} \in P,
\end{aligned}
$$

where

$$
\begin{aligned}
& \mathbf{U}=\mathbf{H}_{b, l}^{1}(\Omega)=\left\{\mathbf{u} \in H^{1}(\Omega)^{2} /\left.\mathbf{u}\right|_{\Gamma_{b} \cup \Gamma_{l}}=0\right\}, \\
& V=H_{z, 0}^{1}(\Omega)=\left\{v \in L^{2}(\Omega) / \partial_{z} v \in L^{2}(\Omega),\left.v\right|_{\Gamma_{s} \cup \Gamma_{b}}=0\right\}, \\
& P=L_{0}^{2}(\Omega)=\left\{p \in L^{2}(\Omega) / \int_{\Omega} p=0\right\} .
\end{aligned}
$$

Moreover, the following energy estimates are shown:

$$
\|\nabla \mathbf{u}\| \leq \frac{1}{\nu}\|\mathbf{f}\|_{\mathbf{U}^{\prime}}, \quad\left\|\partial_{z} v\right\| \leq \frac{1}{\nu \beta_{v}}\|\mathbf{f}\|_{\mathbf{U}^{\prime}}, \quad\|p\| \leq \frac{2}{\beta_{p}}\|\mathbf{f}\|_{\mathbf{U}^{\prime}},
$$

where $\|\cdot\|$ stands for $L^{2}(\Omega)$ norm, $\|\cdot\|_{\mathbf{U}^{\prime}}$ is the norm in the dual space of $\mathbf{U}$ and $\beta_{p}$, $\beta_{v}>0$ are two constants appearing in the following (continuous) inf-sup conditions:

$$
\begin{aligned}
& \sup _{0 \neq(\mathbf{u}, v) \in \mathbf{U} \times V} \frac{(\nabla \cdot(\mathbf{u}, v), p)}{\left\|\left(\nabla \mathbf{u}, \partial_{z} v\right)\right\|} \geq \beta_{p}\|p\| \quad \forall p \in P, \\
& \sup _{0 \neq p \in P} \frac{\left(\partial_{z} v, p\right)}{\|p\|} \geq \beta_{v}\left\|\partial_{z} v\right\| \quad \forall v \in V .
\end{aligned}
$$

Hereafter, for the sake of simplicity, horizontal isotropic (eddy) viscosity coefficient $\nu>0$ is considered. Also, $\left\|\left(\nabla \mathbf{u}, \partial_{z} v\right)\right\|=\sqrt{\|\nabla \mathbf{u}\|^{2}+\left\|\partial_{z} v\right\|^{2}}$ denotes the euclidean norm related to the vectorial space $\mathbf{U} \times V$. 
In $[1,2]$ and [15], this theory is also extended to its discrete counterpart, based on the introduction of "anisotropic" FE approximations of the velocity field, that is, the approximation of each component of the velocity in a different FE space. More specifically, let $\mathbf{U}_{h} \subset \mathbf{U}, V_{h} \subset V$ and $P_{h} \subset P$ be conforming FE spaces and let us consider the following scheme related to (1.14)-(1.16):

$$
\begin{aligned}
\nu\left(\nabla \mathbf{u}_{h}, \nabla \overline{\mathbf{u}}_{h}\right)-\left(p_{h}, \nabla_{\mathbf{x}} \cdot \overline{\mathbf{u}}_{h}\right) & =\left(\mathbf{f}, \overline{\mathbf{u}}_{h}\right) & & \forall \overline{\mathbf{u}}_{h} \in \mathbf{U}_{h}, \\
\left(p_{h}, \partial_{z} \bar{v}_{h}\right) & =0 & & \forall \bar{v}_{h} \in V_{h}, \\
\left(\nabla \cdot\left(\mathbf{u}_{h}, v_{h}\right), \bar{p}_{h}\right) & =0 & & \forall \bar{p}_{h} \in P_{h} .
\end{aligned}
$$

For simplicity, we assume $\mathbf{f}$ in $L^{2}(\Omega)^{2}$.

Let us consider the discrete inf-sup inequalities related to $(I S)^{P}$ and $(I S)^{V}$ : there exits $\gamma_{p}, \gamma_{v}>0$ such that

$$
\begin{array}{lrl}
(I S)_{h}^{P} & \sup _{0 \neq\left(\mathbf{u}_{h}, v_{h}\right) \in \mathbf{U}_{h} \times V_{h}} \frac{\left(\nabla \cdot\left(\mathbf{u}_{h}, v_{h}\right), p_{h}\right)}{\left\|\nabla \mathbf{u}_{h}, \partial_{z} v_{h}\right\|} \geq \gamma_{p}\left\|p_{h}\right\| \quad \forall p_{h} \in P_{h}, \\
(I S)_{h}^{V} & \sup _{\substack{0 \neq p_{h} \in P_{h}\\
}} \frac{\left(p_{h}, \partial_{z} v_{h}\right)}{\left\|p_{h}\right\|} \geq \gamma_{v}\left\|\partial_{z} v_{h}\right\| \quad \forall v_{h} \in V_{h} .
\end{array}
$$

Then, the following two conditions are equivalent:

1. Both $(I S)_{h}^{P}$ and $(I S)_{h}^{V}$ hold.

2. Problem (1.21)-(1.23) is well-posed, i.e., one has the existence and uniqueness of (1.21)-(1.23), and the following energy estimates hold:

$$
\left\|\nabla \mathbf{u}_{h}\right\| \leq \frac{1}{\nu}\|\mathbf{f}\|_{\mathbf{U}^{\prime}}, \quad\left\|\partial_{z} v_{h}\right\| \leq \frac{1}{\nu \gamma_{v}}\|\mathbf{f}\|_{\mathbf{U}^{\prime}}, \quad\left\|p_{h}\right\| \leq \frac{2}{\gamma_{p}}\|\mathbf{f}\|_{\mathbf{U}^{\prime}} .
$$

Note that if $\gamma_{p}$ and $\gamma_{v}$ are independent of $h$, then stability estimates for $\left(\mathbf{u}_{h}, v_{h}, p_{h}\right)$ are obtained in the $\mathbf{U} \times V \times P$-norm. Constraint $(I S)_{h}^{P}$ is similar (and, in fact, weaker) to the well-known Stokes discrete inf-sup or LBB condition (see [6] for more details): there exists $\gamma_{S}>0$ such that

$$
\sup _{0 \neq\left(\mathbf{u}_{h}, v_{h}\right) \in \mathbf{U}_{h} \times V_{h}} \frac{\left(\nabla \cdot\left(\mathbf{u}_{h}, v_{h}\right), p_{h}\right)}{\left\|\nabla \mathbf{u}_{h}\right\|+\left\|\nabla v_{h}\right\|} \geq \gamma_{S}\left\|p_{h}\right\| .
$$

Nevertheless, $(I S)_{h}^{V}$ is a different restriction which has no counterpart in the Stokes framework.

For Taylor-Hood $\mathcal{P}_{2}-\mathcal{P}_{1}$ FE, which is also denoted by $\left(\mathcal{P}_{2}, \mathcal{P}_{2}\right)-\mathcal{P}_{1}$ (i.e., $\mathbf{U}_{h}, V_{h}$, and $P_{h}$ are defined, respectively, by continuous $\mathcal{P}_{2}, \mathcal{P}_{2}$, and $\mathcal{P}_{1}$ FE spaces), it is shown in [15] that $(I S)_{h}^{P}$ holds but $(I S)_{h}^{V}$ does not. This is the reason why the approximation of the anisotropic Navier-Stokes system (1.1)-(1.3) is not stable for small $\varepsilon$ [15]. The same applies to other classical stable $\mathrm{FE}$ like the so-called mini-element $\mathcal{P}_{1, b}-\mathcal{P}_{1}$.

On the other hand, for $\left(\mathcal{P}_{2}, \mathcal{P}_{1}\right)-\mathcal{P}_{1} \mathrm{FE},(I S)_{h}^{P}$ holds in uniformly unstructured meshes, as is proved in [14] applying Stokes stability results about unequal approximations for $\mathbf{U}_{h}$ and $V_{h}$. Numerical simulations suggest that $(I S)_{h}^{V}$ also holds in unstructured meshes and therefore $\left(\mathcal{P}_{2}, \mathcal{P}_{1}\right)-\mathcal{P}_{1}$ would be stable. Similar results were obtained for $\left(\mathcal{P}_{1, b}, \mathcal{P}_{1}\right)-\mathcal{P}_{1}$ (bubble enriching of $\mathbf{U}_{h}$ ) and also some generalizations to $3 \mathrm{D}$ domains; see [14] for more details.

The rest of this paper is organized as follows: In section 2, a reformulation of $(1.21)-(1.23)$ is proposed, introducing the residual term $\left(\nabla \cdot\left(\mathbf{u}_{h}, v_{h}\right), \partial_{z} \bar{v}_{h}\right)$ avoiding 
the restriction $(I S)_{h}^{V}$. Then well-posedness and error estimates of this reformulated problem are shown (using a saddle-point framework [6]) only requiring that $\mathbf{U}_{h}, V_{h}$, and $P_{h}$ satisfy the Stokes-like stability restriction $(I S)_{h}^{P}$. Moreover, $O\left(h^{2}\right)$ accuracy is proved for $\mathcal{P}_{2}-\mathcal{P}_{1}$ and order $O(h)$ for $\mathcal{P}_{1, b}-\mathcal{P}_{1}$.

In section 3 , previous reformulation is extended, adding to (1.23) the consistent term $\left(\partial_{z} p, \partial_{z} \bar{p}\right)$. Again, well-posedness and error estimates are shown (using a vectorial approach laying in a generalized Lax-Milgram theorem), where $p_{h}$ is controlled in a stronger norm (with $\partial_{z} p_{h} \in L^{2}(\Omega)$ ). $O(h)$ accuracy is proved again for $\mathcal{P}_{1, b}-\mathcal{P}_{1}$, but also including $\partial_{z} p_{h}$ in $L^{2}(\Omega)$. For $\mathcal{P}_{2}-\mathcal{P}_{1}$, it is not clear the $O\left(h^{2}\right)$ accuracy for all meshes.

Some numerical tests are performed in section 4. The first one shows the applicability of this new scheme to approach the primitive equations in a domain built from real data of bathymetry. The particular area of the Gibraltar Strait is considered, in two dimensions, although these experiments can easily be extended to 3D oceanic regions. Finally, we provide some numerical tests which conform this paper's theoretical results about stability and accuracy rates.

2. Stabilization of vertical velocity. Let us consider the following reformulation of (1.14)-(1.16): find $(\mathbf{u}, v, p) \in \mathbf{U} \times V \times P$ (spaces defined in (1.17)-(1.19)) such that

$$
\begin{aligned}
\nu(\nabla \mathbf{u}, \nabla \overline{\mathbf{u}})-\left(p, \nabla_{\mathbf{x}} \cdot \overline{\mathbf{u}}\right) & =\langle\mathbf{f}, \overline{\mathbf{u}}\rangle & & \forall \overline{\mathbf{u}} \in \mathbf{U}, \\
\nu\left(\nabla \cdot(\mathbf{u}, v), \partial_{z} \bar{v}\right)-\left(p, \partial_{z} \bar{v}\right) & =0 & & \forall \bar{v} \in V, \\
(\nabla \cdot(\mathbf{u}, v), \bar{p}) & =0 & & \forall \bar{p} \in P .
\end{aligned}
$$

This new system is obtained by adding to (1.15) the consistent term $\nu(\nabla$. $\left.(\mathbf{u}, v), \partial_{z} \bar{v}\right)$ (which vanishes in the continuous problem). Indeed, (1.16) or (2.3) imply $\nabla \cdot(\mathbf{u}, v)=0$ almost everywhere in $\Omega$, and hence system (2.1)-(2.3) coincides with (1.14)-(1.16). Therefore, there is a unique solution of (2.1)-(2.3) which, in particular, satisfies the energy estimates (1.20).

In the discrete case, let $\mathbf{U}_{h} \subset \mathbf{U}, V_{h} \subset V$, and $P_{h} \subset P$ be three conforming $\mathrm{FE}$ spaces and let us consider the following scheme: find $\mathbf{u}_{h} \in \mathbf{U}_{h}, v_{h} \in V_{h}$, and $p_{h} \in P_{h}$ solving

$$
\begin{aligned}
\nu\left(\nabla \mathbf{u}_{h}, \nabla \overline{\mathbf{u}}_{h}\right)-\left(p_{h}, \nabla_{\mathbf{x}} \cdot \overline{\mathbf{u}}_{h}\right) & =\left\langle\mathbf{f}, \overline{\mathbf{u}}_{h}\right\rangle & & \forall \overline{\mathbf{u}}_{h} \in \mathbf{U}_{h}, \\
\nu\left(\nabla \cdot\left(\mathbf{u}_{h}, v_{h}\right), \partial_{z} \bar{v}_{h}\right)-\left(p_{h}, \partial_{z} \bar{v}_{h}\right) & =0 & & \forall \bar{v}_{h} \in V_{h}, \\
\left(\nabla \cdot\left(\mathbf{u}_{h}, v_{h}\right), \bar{p}_{h}\right) & =0 & & \forall \bar{p}_{h} \in P_{h} .
\end{aligned}
$$

Note that if the condition $\partial_{z} V_{h} \subset P_{h}$ is satisfied, then $\left(\nabla \cdot\left(\mathbf{u}_{h}, v_{h}\right), \partial_{z} \bar{v}_{h}\right)=0$ according to (2.6) and schemes (1.21)-(1.23) and (2.4)-(2.6) are equivalent. This occurs, for instance, when $V_{h}$ and $P_{h}$ are defined by $\mathcal{P}_{1}$ and $\mathcal{P}_{0}$, respectively. Moreover, $(I S)_{h}^{V}$ is easily satisfied when $\partial_{z} V_{h} \subset P_{h}$, and hence the stability of schemes holds only imposing the discrete Stokes-like stability constraint $(I S)_{h}^{P}$.

Although the equivalence of both schemes cannot be assured if $\partial_{z} V_{h} \not \subset P_{h}$, we are going to show that $(I S)_{h}^{P}$ is also a sufficient condition for the well-posedness of (2.4)-(2.6), i.e., the discrete hydrostatic stability constraint $(I S)_{h}^{V}$ is not necessary when (1.15) is reformulated as (2.5). In particular, any standard LBB-stable FE will be stable for (2.4)-(2.6). We also provide stability and error estimates for this scheme.

2.1. Generic saddle-point framework. We start summarizing on some wellknown results from the saddle-point theory for mixed $\mathrm{FE}$ (see, e.g., $[6,5]$ ). Let $\mathbf{W}$ 
and $P$ be two Hilbert spaces, let $a(\cdot, \cdot)$ be a continuous bilinear form on $\mathbf{W} \times \mathbf{W}$, and let $b(\cdot, \cdot)$ be a continuous bilinear form on $\mathbf{W} \times P$, denoting by $\|a\|$ and $\|b\|$ their respective continuity constants. We consider the following mixed problem:

$$
\begin{array}{rlrl}
a(\mathbf{w}, \overline{\mathbf{w}})+b(p, \overline{\mathbf{w}}) & =\langle\mathbf{F}, \overline{\mathbf{w}}\rangle_{\mathbf{W}^{\prime} \times \mathbf{W}} & & \forall \overline{\mathbf{w}} \in \mathbf{W}, \\
b(\bar{p}, \mathbf{w}) & =0 & \forall \bar{p} \in P,
\end{array}
$$

where $\mathbf{F} \in \mathbf{W}^{\prime}$. We denote by $B: \mathbf{W} \rightarrow P^{\prime}$ and $B^{t}: P \rightarrow \mathbf{W}^{\prime}$ the linear forms defined as $\langle B \mathbf{w}, p\rangle=b(\mathbf{w}, p)=\left\langle\mathbf{w}, B^{t} p\right\rangle$ for all $\mathbf{w} \in \mathbf{W}, p \in P$. From mixed methods theory (see, for instance, [6, Propositions 1.1 and 1.3], and [15, Lenma 4]) one has well posedness of $(2.7)-(2.8)$ if

- $a(\cdot, \cdot)$ is coercive on $\operatorname{ker} B$, that is, there exists $\alpha>0$ such that

$$
a(\mathbf{w}, \mathbf{w}) \geq \alpha\|\mathbf{w}\|_{\mathbf{w}}^{2} \quad \forall \mathbf{w} \in \operatorname{ker} B,
$$

- and $b(\cdot, \cdot)$ verifies an inf-sup constion, i.e., there exists $\gamma>0$ such that

$$
\sup _{\mathbf{w} \in \mathbf{W}} \frac{b(\mathbf{w}, p)}{\|\mathbf{w}\| \mathbf{w}} \geq \gamma\|p\|_{P / \operatorname{ker} B^{t}} \quad \forall p \in P .
$$

For the discrete case, let us consider two finite-dimensional subspaces $\mathbf{W}_{h} \subset \mathbf{W}$, $P_{h} \subset P$ and the linear forms $B_{h}: \mathbf{W}_{h} \rightarrow P_{h}^{\prime}, B_{h}^{t}: P_{h} \rightarrow \mathbf{W}_{h}^{\prime}$, defined as $\left\langle B_{h} \mathbf{w}_{h}, p_{h}\right\rangle=$ $b\left(\mathbf{w}_{h}, p_{h}\right)=\left\langle\mathbf{w}_{h}, B_{h}^{t} p_{h}\right\rangle$ for all $\mathbf{w}_{h} \in \mathbf{W}_{h}, p_{h} \in P_{h}$. The following result provides error estimates for the solution of the discrete mixed problem: find $\left(\mathbf{w}_{h}, p_{h}\right) \in \mathbf{W}_{h} \times P_{h}$ such that

$$
\begin{array}{rlrl}
a\left(\mathbf{w}_{h}, \overline{\mathbf{w}}_{h}\right)+b\left(p_{h}, \overline{\mathbf{w}}_{h}\right) & =\left\langle\mathbf{F}, \overline{\mathbf{w}}_{h}\right\rangle_{\mathbf{W}^{\prime}, \mathbf{W}} & & \forall \overline{\mathbf{w}}_{h} \in \mathbf{W}_{h}, \\
b\left(\bar{p}_{h}, \mathbf{w}_{h}\right) & =0 & \forall \bar{p}_{h} \in P_{h} .
\end{array}
$$

Lemma 2.1. Assuming previous conditions for well-posedness, let $(\mathbf{w}, p)$ be the solution of problem (2.7)-(2.8). If the following two conditions hold,

1. there exists $\alpha>0$ such that

$$
a\left(\mathbf{w}_{h}, \mathbf{w}_{h}\right) \geq \alpha\left\|\mathbf{w}_{h}\right\|_{\mathbf{w}}^{2} \quad \forall \mathbf{w}_{h} \in \operatorname{ker} B_{h},
$$

2. there exists $\gamma>0$ such that

$$
\sup _{\mathbf{w}_{h} \in \mathbf{W}_{h}} \frac{b\left(\mathbf{w}_{h}, p_{h}\right)}{\left\|\mathbf{w}_{h}\right\|_{\mathbf{w}}} \geq \gamma\left\|p_{h}\right\|_{P / \operatorname{ker} B_{h}^{t}} \quad \forall p_{h} \in P_{h},
$$

then there exists a unique solution $\left(\mathbf{w}_{h}, p_{h}\right)$ of (2.9)-(2.10) which satisfies

$$
\left\|\mathbf{w}_{h}\right\|_{\mathbf{w}} \leq \frac{1}{\alpha}\|\mathbf{f}\|_{\mathbf{W}^{\prime}}, \quad\left\|p_{h}\right\|_{P / \operatorname{ker} B^{t}} \leq \frac{1}{\gamma}\left(1+\frac{\|a\|}{\alpha}\right)\|\mathbf{f}\|_{\mathbf{W}^{\prime}} .
$$

If $\alpha$ and $\gamma$ are independent of $h$, then there exist four constants $C_{1}, C_{2}, C_{3}$, and $C_{4}$, independent of $h$, such that $\left(\mathbf{w}_{h}, p_{h}\right)$ satisfies

$$
\begin{aligned}
\left\|\mathbf{w}-\mathbf{w}_{h}\right\|_{\mathbf{w}} & \leq C_{1} \inf _{\overline{\mathbf{w}}_{h} \in \mathbf{W}_{h}}\left\|\mathbf{w}-\overline{\mathbf{w}}_{h}\right\|_{\mathbf{w}}+C_{2} \inf _{\bar{p}_{h} \in P_{h}}\left\|p-\bar{p}_{h}\right\|_{P}, \\
\left\|p-p_{h}\right\|_{P} & \leq C_{3} \inf _{\overline{\mathbf{w}}_{h} \in \mathbf{W}_{h}}\left\|\mathbf{w}-\overline{\mathbf{w}}_{h}\right\|_{\mathbf{w}}+C_{4} \inf _{\bar{p}_{h} \in P_{h}}\left\|p-\bar{p}_{h}\right\|_{P} .
\end{aligned}
$$

Moreover, constants $C_{1}, C_{2}, C_{3}$, and $C_{4}$ satisfy

$$
\begin{array}{ll}
C_{1} \leq\left(1+\frac{\|a\|}{\alpha}\right)\left(1+\frac{\|b\|}{\gamma}\right), & C_{2} \leq \frac{\|b\|}{\alpha} \\
C_{3} \leq \frac{\|a\|}{\gamma}\left(1+\frac{\|a\|}{\alpha}\right)\left(1+\frac{\|b\|}{\gamma}\right), & C_{4} \leq 1+\frac{\|b\|}{\gamma}+\frac{\|a\|\|b\|}{\alpha \gamma} .
\end{array}
$$

Copyright $@$ by SIAM. Unauthorized reproduction of this article is prohibited. 
2.2. Application to $\boldsymbol{v}$-stabilized formulation. Our problems (2.1)-(2.3) and (2.4)-(2.6) can be written, respectively, in the generic forms (2.7)-(2.8) and (2.9)(2.10). For this purpose, it is sufficient to consider the Hilbert space $\mathbf{W}=\mathbf{U} \times V$, where $\mathbf{U}$ and $V$ are defined in (1.17) and (1.18), endowed with the following inner product and norm:

$$
(\mathbf{w}, \overline{\mathbf{w}})_{\mathbf{W}}=(\nabla \mathbf{u}, \nabla \overline{\mathbf{u}})+\left(\partial_{z} v, \partial_{z} \bar{v}\right), \quad\|\mathbf{w}\|_{\mathbf{W}}^{2}=\|\nabla \mathbf{u}\|^{2}+\left\|\partial_{z} v\right\|^{2}
$$

for all $\mathbf{w}=(\mathbf{u}, v)$ and $\overline{\mathbf{w}}=(\overline{\mathbf{u}}, \bar{v}) \in \mathbf{W}$. We also consider the space $P$, defined in (1.19) with the usual $L^{2}(\Omega)$ scalar product and the following bilinear and linear forms:

$$
\begin{aligned}
a(\mathbf{w}, \overline{\mathbf{w}}) & :=\nu(\nabla \mathbf{u}, \nabla \overline{\mathbf{u}})+\nu\left(\partial_{z} v, \partial_{z} \bar{v}\right)+\nu\left(\nabla_{\mathbf{x}} \cdot \mathbf{u}, \partial_{z} \bar{v}\right), \\
b(p, \overline{\mathbf{w}}) & :=-\left(p, \nabla_{\mathbf{x}} \cdot \overline{\mathbf{u}}\right)-\left(p, \partial_{z} \bar{v}\right), \\
\langle\mathbf{F}, \overline{\mathbf{w}}\rangle_{\mathbf{W}^{\prime}, \mathbf{W}} & :=\langle(\mathbf{f}, 0), \overline{\mathbf{w}}\rangle_{\mathbf{W}^{\prime}, \mathbf{W}}=\langle\mathbf{f}, \overline{\mathbf{u}}\rangle_{\mathbf{U}^{\prime}, \mathbf{U}}
\end{aligned}
$$

for each $\mathbf{w}=(\mathbf{u}, v), \overline{\mathbf{w}}=(\overline{\mathbf{u}}, \bar{v}) \in \mathbf{W}$, and $p \in P$.

Remark 1 . The form $a(\cdot, \cdot)$ is not symmetric, due to the stabilization term. A symmetric bilinear form can be defined, introducing the consistent term $-\nu \nabla_{\mathbf{x}}(\nabla \cdot(\mathbf{u}, v))$ in the horizontal momentum equation (2.1) (where $\nabla_{\mathbf{x}}=\left(\partial_{x}, \partial_{y}\right)^{t}$ is the horizontal gradient), arriving at

$$
\nu(\nabla \mathbf{u}, \nabla \overline{\mathbf{u}})+\nu\left(\nabla_{\mathbf{x}} \cdot \mathbf{u}, \nabla_{\mathbf{x}} \cdot \overline{\mathbf{u}}\right)+\nu\left(\partial_{z} v, \nabla_{\mathbf{x}} \cdot \overline{\mathbf{u}}\right)-\left(p, \nabla_{\mathbf{x}} \cdot \overline{\mathbf{u}}\right)=\langle\mathbf{f}, \overline{\mathbf{u}}\rangle \quad \forall \overline{\mathbf{u}} \in \mathbf{U} .
$$

Hence, the symmetric bilinear form reads

$$
\begin{aligned}
\widehat{a}(\mathbf{w}, \overline{\mathbf{w}}):= & \nu(\nabla \mathbf{u}, \nabla \overline{\mathbf{u}})+\nu\left(\nabla_{\mathbf{x}} \cdot \mathbf{u}, \nabla_{\mathbf{x}} \cdot \overline{\mathbf{u}}\right)+\nu\left(\partial_{z} v, \nabla_{\mathbf{x}} \cdot \overline{\mathbf{u}}\right) \\
& +\nu\left(\partial_{z} v, \partial_{z} \bar{v}\right)+\nu\left(\nabla_{\mathbf{x}} \cdot \mathbf{u}, \partial_{z} \bar{v}\right) .
\end{aligned}
$$

See Remark 2 for a proof of the coercivity of $\widehat{a}$.

Now, well-posedness and the stability of the discrete problem (2.4)-(2.6) can be shown.

Theorem 2.2 (stability). Let $\mathbf{U}_{h} \subset \mathbf{U}, V_{h} \subset V$ and $P_{h} \subset P$ be families of FE in a regular partition $\mathcal{T}_{h}$ of $\Omega$ satisfying the inf-sup condition $(I S)_{h}^{P}$. Then scheme (2.4)(2.6) has a unique solution $\left(\mathbf{u}_{h}, v_{h}, p_{h}\right) \in \mathbf{U}_{h} \times V_{h} \times P_{h}$, which satisfies the following stability estimates:

$$
\left\|\nabla \mathbf{u}_{h}\right\|^{2}+\left\|\partial_{z} v_{h}\right\|^{2} \leq \frac{4}{\nu^{2}}\|\mathbf{f}\|_{\mathbf{U}^{\prime}}^{2}, \quad\left\|p_{h}\right\| \leq \frac{5}{\gamma_{p}}\|\mathbf{f}\|_{\mathbf{U}^{\prime}}
$$

where $\gamma_{p}$ is the constant given in $(I S)_{h}^{P}$.

Proof. Let $\mathbf{W}_{h}=\mathbf{U}_{h} \times V_{h}$. For the existence and uniqueness of (2.4)-(2.6), it is sufficient to show that the bilinear forms $a(\cdot, \cdot)$ and $b(\cdot, \cdot)$, defined in (2.14) and (2.15) satisfy, respectively, the coercivity and inf-sup conditions (2.11) and (2.12).

For the coercivity of $a(\cdot, \cdot)$ in $\mathbf{W}_{h}$, using the following technical result (see [15, Lemma 4]),

$$
\left\|\nabla_{\mathbf{x}} \cdot \mathbf{u}\right\| \leq\left\|\nabla_{\mathbf{x}} \mathbf{u}\right\| \quad \forall \mathbf{u} \in \mathbf{H}_{b, l}^{1}(\Omega),
$$

one has

$$
\begin{aligned}
a\left(\mathbf{w}_{h}, \mathbf{w}_{h}\right) & =\nu\left\|\nabla \mathbf{u}_{h}\right\|^{2}+\nu\left(\nabla_{\mathbf{x}} \cdot \mathbf{u}_{h}, \partial_{z} v_{h}\right)+\nu\left\|\partial_{z} v_{h}\right\|^{2} \\
& \geq \nu\left\|\nabla \mathbf{u}_{h}\right\|^{2}-\frac{\nu}{2}\left\|\nabla_{\mathbf{x}} \cdot \mathbf{u}_{h}\right\|^{2}-\frac{\nu}{2}\left\|\partial_{z} v\right\|^{2}+\nu\left\|\partial_{z} v_{h}\right\|^{2} \\
& \geq \frac{\nu}{2}\left(\left\|\nabla \mathbf{u}_{h}\right\|^{2}+\left\|\partial_{z} v_{h}\right\|^{2}\right) .
\end{aligned}
$$

Copyright $\odot$ by SIAM. Unauthorized reproduction of this article is prohibited. 
On the other hand, if $(I S)_{h}^{P}$ holds, inf-sup condition (2.1) is satisfied for $\beta=\gamma_{p}$. (Note that $(I S)_{h}^{P}$ imply ker $B_{h}^{t}=\{0\}$, and therefore $\|\cdot\|_{P / \text { ker }} B_{h}^{t}$ can be identified with the $L^{2}(\Omega)$-norm.) Therefore, system (2.4)-(2.6) has a unique solution in $\mathbf{W}_{h} \times P_{h}$. Also, the stability inequalities (2.13) hold, which implies (2.19) (taking into account that, from (2.14), $\|a\|=2 \nu$ and, from (2.21), $\alpha=\nu / 2$ ).

By using the saddle-point framework, error estimates can also be obtained.

TheOREM 2.3 (error estimates). Under the conditions of Theorem 2.2 , let $(\mathbf{w}, p)=$ $(\mathbf{u}, v, p)$ be the solution of problem (1.14)-(1.16) (or (2.1)-(2.3)) and let $\left(\mathbf{w}_{h}, p_{h}\right)=$ $\left(\mathbf{u}_{h}, v_{h}, p_{h}\right)$ be the solution of scheme (2.4)-(2.6). Assume that there exists a positive constant $\gamma_{p}>0$ satisfying $(I S)_{h}^{P}$. Then

$$
\begin{aligned}
\left\|\mathbf{w}-\mathbf{w}_{h}\right\|_{\mathbf{w}} & \leq C_{1} \inf _{\overline{\mathbf{w}}_{h} \in \mathbf{W}_{h}}\left\|\mathbf{w}-\overline{\mathbf{w}}_{h}\right\|_{\mathbf{w}}+C_{2} \inf _{\bar{p}_{h} \in P_{h}}\left\|p-\bar{p}_{h}\right\|_{P}, \\
\left\|p-p_{h}\right\|_{P} & \leq C_{3} \inf _{\overline{\mathbf{w}}_{h} \in \mathbf{W}_{h}}\left\|\mathbf{w}-\overline{\mathbf{w}}_{h}\right\|_{\mathbf{w}}+C_{4} \inf _{\bar{p}_{h} \in P_{h}}\left\|p-\bar{p}_{h}\right\|_{P},
\end{aligned}
$$

where

$$
C_{1} \leq 5\left(1+\frac{1}{\gamma_{p}}\right), \quad C_{2} \leq \frac{2}{\nu}, \quad C_{3} \leq \frac{10 \nu}{\gamma_{p}}\left(1+\frac{1}{\gamma_{p}}\right), \quad C_{4} \leq 1+\frac{5}{\gamma_{p}} .
$$

Proof. Problems (2.1)-(2.3) and (2.4)-(2.6) can be reformulated as (2.7)-(2.8) and (2.9)-(2.10), respectively, where the bilinear and linear forms are defined in (2.14)(2.16). Therefore, (2.22)-(2.23) can be obtained from Lemma 2.1, taking $\|a\|=2 \nu$, $\|b\|=1, \alpha=\nu / 2$, and $\gamma=\gamma_{p}$.

Remark 2. In the symmetric case (see Remark 1), the coercivity of the bilinear form $\widehat{a}$ can also be shown, and hence a stability result, similar to Theorem 2.2, can be obtained for the symmetric reformulation (2.17)-(2.2)-(2.3). Indeed, applying the Young inequality $2 a b \leq \frac{3}{2} a^{2}+\frac{2}{3} b^{2}$ and (2.20),

$$
\begin{aligned}
\widehat{a}\left(\mathbf{w}_{h}, \mathbf{w}_{h}\right) & =\nu\left\|\nabla \mathbf{u}_{h}\right\|^{2}+\nu\left\|\nabla_{\mathbf{x}} \cdot \mathbf{u}_{h}\right\|^{2}+\nu\left\|\partial_{z} v_{h}\right\|^{2}+2 \nu\left(\nabla_{\mathbf{x}} \cdot \mathbf{u}_{h}, \partial_{z} v_{h}\right) \\
& \geq \nu\left\|\nabla \mathbf{u}_{h}\right\|^{2}+\nu\left\|\nabla_{\mathbf{x}} \cdot \mathbf{u}_{h}\right\|^{2}+\nu\left\|\partial_{z} v_{h}\right\|^{2}-\frac{3 \nu}{2}\left\|\nabla_{\mathbf{x}} \cdot \mathbf{u}_{h}\right\|^{2}-\frac{2 \nu}{3}\left\|\partial_{z} v_{h}\right\| \\
& \geq \frac{\nu}{2}\left\|\nabla \mathbf{u}_{h}\right\|^{2}+\frac{\nu}{3}\left\|\partial_{z} v_{h}\right\|^{2} .
\end{aligned}
$$

Note that, in this symmetric case, the coercivity coefficient for the $v$ component is slightly lower than in nonsymmetric one, passing from $\nu / 2$ to $\nu / 3$. Finally, error estimates similar to Theorem 2.3 can also be obtained for the symmetric reformulation.

Remark 3 (about convergence orders). Theorem 2.3 allows obtaining convergence results, using the standard FE interpolation theory. For example, let $r \geq 1$ and let $\mathcal{P}_{r}$ denote $C^{0}$ piecewise polynomials of degree $\leq r$ in a nondegenerated triangulation of a polygonal domain $\Omega$ with maximum triangle diameter $h$. It is well known (see, e.g., [5, Corollary 4.2.4]) that if $\mathcal{I}^{h} \phi$ denotes the FE nodal interpolation of $\phi$ in $\mathcal{P}_{r}$, then

$$
\left\|\phi-\mathcal{I}^{h} \phi\right\|_{H^{s}} \leq C h^{r-s+1}\|\phi\|_{H^{r+1}}
$$

for all $\phi \in H^{r+1}(\Omega)$ and $0 \leq s \leq r$.

By using Theorem 2.3 and applying (2.24), one can deduce some convergence estimates. For example, assuming that both $\mathbf{u}_{h}$ and $v_{h}$ are approximated in the same space, $\mathcal{P}_{r}(r \geq 2)$, and $p_{h}$ is approximated in $\mathcal{P}_{r-1}$, we have

$$
\left\|\nabla\left(\mathbf{u}-\mathbf{u}_{h}\right)\right\|+\left\|\partial_{z}\left(v-v_{h}\right)\right\|+\left\|p-p_{h}\right\| \leq C h^{r}\left(\|(\mathbf{u}, v)\|_{H^{r+1}(\Omega)^{d}}+\|p\|_{H^{r}(\Omega)}\right) .
$$


In particular, order $O\left(h^{2}\right)$ is obtained in the $\mathcal{P}_{2}-\mathcal{P}_{1}$ case if $(\mathbf{u}, v) \in H^{3}(\Omega)$ and $p \in H^{2}(\Omega)$. In a similar way, order $O(h)$ could also be obtained for the combination $\mathcal{P}_{1, b}-\mathcal{P}_{1}$ if $(\mathbf{u}, v) \in H^{2}(\Omega)$ and $p \in H^{1}(\Omega)$. Our numerical results agree with this statement, as can be seen in Test 3 below.

On the other hand, if $\mathbf{u}_{h}$ and $v_{h}$ are approximated in different spaces, $\mathcal{P}_{r_{1}}$ and $\mathcal{P}_{r_{2}}$, only order $O\left(h^{r}\right)$ with $r=\min \left(r_{1}, r_{2}\right)$ can be obtained from Theorem 2.3. Anyway, numerical tests in literature show that better results can be expected; for example, for the nonstabilized scheme $(1.21)-(1.23)$ with the $\left(\mathcal{P}_{2}, \mathcal{P}_{1}\right)-\mathcal{P}_{1} \mathrm{FE}$, order $O\left(h^{1.5}\right)$ for $\mathbf{u}_{h}$ is obtained in the numerical experiments given in [15].

Finally, note that error estimate (2.25) evolves different norms for $\mathbf{u}-\mathbf{u}_{h}$ and $v-v_{h}$, but the isotropic regularity $\|(\mathbf{u}, v)\|_{H^{r+1}(\Omega)^{d}}$ is required due to (2.24).

2.3. Stabilization of the anisotropic (nonhydrostatic) problem. The theory developed in sections 2.1 and 2.2 can be extended for the approximation of more general problems, where the hydrostatic constraint $\partial_{z} p=0$ is relaxed by $-\varepsilon^{2} \Delta v+\partial_{z} p=0$ and the extra boundary conditions for $v$ given in (1.6).

Let us consider the following variational problem arising from the problem (1.1)(1.3) plus the consistent stabilizing term $\nu\left(\nabla \cdot(\mathbf{u}, v), \partial_{z} \bar{v}\right)$ :

$$
\begin{aligned}
\nu(\nabla \mathbf{u}, \nabla \overline{\mathbf{u}})-\left(p, \nabla_{\mathbf{x}} \cdot \overline{\mathbf{u}}\right) & =\langle\mathbf{f}, \overline{\mathbf{u}}\rangle & & \forall \overline{\mathbf{u}} \in \mathbf{U}, \\
\varepsilon^{2}(\nabla v, \nabla \bar{v})+\nu\left(\nabla \cdot(\mathbf{u}, v), \partial_{z} \bar{v}\right)-\left(p, \partial_{z} \bar{v}\right) & =0 & & \forall \bar{v} \in V, \\
(\nabla \cdot(\mathbf{u}, v), \bar{p}) & =0 & & \forall \bar{p} \in P .
\end{aligned}
$$

The existence and uniqueness of solution of (2.26)-(2.28) holds, because this problem is only a reformulation of the anisotropic Stokes problem (1.1)-(1.3).

The problem (2.26)-(2.28) can be set in the saddle-point framework (2.7)-(2.8) by defining

$$
a(\mathbf{w}, \overline{\mathbf{w}}):=\nu(\nabla \mathbf{u}, \nabla \overline{\mathbf{u}})+\varepsilon^{2}(\nabla v, \nabla \bar{v})+\nu\left(\partial_{z} v, \partial_{z} \bar{v}\right)+\nu\left(\nabla_{\mathbf{x}} \cdot \mathbf{u}, \partial_{z} \bar{v}\right)
$$

for each $\mathbf{w}=(\mathbf{u}, v), \overline{\mathbf{w}}=(\overline{\mathbf{u}}, \bar{v}) \in \mathbf{W}$, and $p \in P$ with $b(\cdot, \cdot)$ and $\langle F, \cdot\rangle$ given in (2.15) and (2.16). Arguing like in section 2.2, stability and error estimates can be deduced for the nonhydrostatic scheme:

$$
\begin{array}{rlrl}
\nu\left(\nabla \mathbf{u}_{h}, \nabla \overline{\mathbf{u}}_{h}\right)-\left(p_{h}, \nabla_{\mathbf{x}} \cdot \overline{\mathbf{u}}_{h}\right) & =\left\langle\mathbf{f}, \overline{\mathbf{u}}_{h}\right\rangle & & \forall \overline{\mathbf{u}}_{h} \in \mathbf{U}_{h}, \\
\varepsilon^{2}\left(\nabla v_{h}, \nabla \bar{v}_{h}\right)+\nu\left(\nabla \cdot\left(\mathbf{u}_{h}, v_{h}\right), \partial_{z} \bar{v}_{h}\right)-\left(p_{h}, \partial_{z} \bar{v}_{h}\right)=0 & & \forall \bar{v}_{h} \in V_{h}, \\
\left(\nabla \cdot\left(\mathbf{u}_{h}, v_{h}\right), \bar{p}_{h}\right) & =0 & & \forall \bar{p}_{h} \in P_{h} .
\end{array}
$$

The former statements can be summarized as follows.

Proposition 2.4. Theorems 2.2 and 2.3 can be extended for scheme (2.30)-(2.32) for $\varepsilon \geq 0$.

3. Regularization of vertical derivative of pressure. The purpose of this section is to approach the hydrostatic Stokes equations (1.14)-(1.16), obtaining an accuracy rate also for the $L^{2}(\Omega)$-norm of $\partial_{z} p$. We start introducing additional consistent terms to the stabilized problem (2.1)-(2.3), where only the Stokes-like inf-sup condition $(I S)^{P}$ must be imposed. Afterward, we analyze the possibility of extending the results to the original (nonstabilized) hydrostatic Stokes equations (1.14)-(1.16). In this case, we will find an unstable scheme.

Let us consider the additional pressure space

$$
\widehat{P}=H_{z}^{1}(\Omega) \cap L_{0}^{2}(\Omega)=\left\{p \in L_{0}^{2}(\Omega) / \partial_{z} p \in L^{2}(\Omega)\right\},
$$


endowed with the norm $\|p\|_{\widehat{P}}^{2}=\|p\|^{2}+\left\|\partial_{z} p\right\|^{2}$. Then, the following reformulation or (2.1)-(2.3) can be considered: find $(\mathbf{u}, v) \in \mathbf{W}=\mathbf{U} \times V=\mathbf{H}_{b, l}^{1}(\Omega) \times H_{z, 0}^{1}(\Omega)$ and $p \in \widehat{P}$ such that

$$
\begin{aligned}
\nu(\nabla \mathbf{u}, \nabla \overline{\mathbf{u}})-\left(p, \nabla_{\mathbf{x}} \cdot \overline{\mathbf{u}}\right) & =(\mathbf{f}, \overline{\mathbf{u}}) & & \forall \overline{\mathbf{u}} \in \mathbf{U}, \\
\nu\left(\nabla \cdot(\mathbf{u}, v), \partial_{z} \bar{v}\right)-\left(p, \partial_{z} \bar{v}\right) & =0 & & \forall \bar{v} \in V, \\
(\nabla \cdot(\mathbf{u}, v), \bar{p})+\left(\partial_{z} p, \partial_{z} \bar{p}\right) & =0 & & \forall \bar{p} \in \widehat{P} .
\end{aligned}
$$

This system is obtained by adding to $(2.3)$ the term $\left(\partial_{z} p, \partial_{z} \bar{p}\right)$, which is consistent in the sense that it vanishes if $p$ satisfies (1.15). Indeed, by a density argument, (1.15) implies $\partial_{z} p=0$ almost everywhere in $\Omega$, and hence it is clear that the solution of (2.1)(2.3) satisfies (3.2)-(3.4). Since we are going to prove the uniqueness of the solution of problem (3.2)-(3.4) (see Theorem 3.1 below), both problems are equivalents. In particular, the solution of (3.2)-(3.4) satisfies the energy estimates (1.20). But another estimate, also involving the $L^{2}(\Omega)$-norm of $\partial_{z} p$, will be provided.

Remark 4. In the anisotropic case (with $\varepsilon>0$ ), reformulation like (3.2)-(3.4) suffers an important modification, because (3.3) must be replaced by

$$
(\nabla \cdot(\mathbf{u}, v), \bar{p})+\left(\partial_{z} p-\varepsilon^{2} \Delta v, \partial_{z} \bar{p}\right)=0 \quad \forall \bar{p} \in \widehat{P},
$$

whose treatment is not straightforward and is not addressed in the current work.

3.1. Well-posedness of the continuous problem. Now, the saddle-point approach used in section 2 can not be employed, because it is not obvious how to obtain inf-sup conditions involving the $H_{z}^{1}$-norm of the pressure space $\widehat{P}$ defined in (3.1). Instead of it, a vectorial approach will be used. Let the vectorial space $\mathbf{X}=\mathbf{W} \times \widehat{P}$ endowed with the norm

$$
\|\chi\|_{\mathbf{X}}=\|(\mathbf{u}, v, p)\|_{\mathbf{x}}=\sqrt{\|\nabla \mathbf{u}\|^{2}+\left\|\partial_{z} v\right\|^{2}+\|p\|^{2}+\left\|\partial_{z} p\right\|^{2}}
$$

(denoted in the following lines by $\left.\left\|\nabla \mathbf{u}, \partial_{z} \mathbf{u}, p, \partial_{z} p\right\|\right)$ for each $\chi=(\mathbf{w}, p) \in \mathbf{X}$ with $\mathbf{w}=(\mathbf{u}, v)$. Let us define the following (nonsymmetric) bilinear form on $\mathbf{X} \times \mathbf{X}$ :

$$
\begin{aligned}
\widetilde{\mathcal{A}}(\boldsymbol{\chi}, \overline{\boldsymbol{\chi}}):= & \nu(\nabla \mathbf{u}, \nabla \overline{\mathbf{u}})+\nu\left(\partial_{z} v, \partial_{z} \bar{v}\right)+\left(\partial_{z} p, \partial_{z} \bar{p}\right) \\
& +\nu\left(\nabla_{\mathbf{x}} \cdot \mathbf{u}, \partial_{z} \bar{v}\right)-(p, \nabla \cdot(\overline{\mathbf{u}}, \bar{v}))+(\nabla \cdot(\mathbf{u}, v), \bar{p}),
\end{aligned}
$$

where $\bar{\chi}=(\overline{\mathbf{w}}, \bar{p}) \in \mathbf{X}$ with $\overline{\mathbf{w}}=(\overline{\mathbf{u}}, \bar{v})$. Then problem (3.2)-(3.4) can be written as

$$
\text { Find } \chi \in \mathbf{X} \text { such that } \widetilde{\mathcal{A}}(\chi, \bar{\chi})=\langle\mathbf{F}, \bar{\chi}\rangle_{\mathbf{X}^{\prime}, \mathbf{X}} \quad \forall \bar{\chi} \in \mathbf{X},
$$

where $\langle\cdot, \cdot \cdot\rangle_{\mathbf{X}^{\prime}, \mathbf{X}}$, denotes the duality product and

$$
\langle\mathbf{F}, \bar{\chi}\rangle_{\mathbf{X}^{\prime}, \mathbf{X}}:=\langle(\mathbf{f}, 0,0), \bar{\chi}\rangle_{\mathbf{X}^{\prime}, \mathbf{X}}=\langle\mathbf{f}, \overline{\mathbf{u}}\rangle_{\mathbf{U}^{\prime}, \mathbf{U}} .
$$

To prove the existence and uniqueness of weak solutions of (3.2)-(3.4), or equivalently (3.6), we can use the following generalized Lax-Milgram theorem (see, for instance, [11, Theorem 2.6]).

Theorem 3.1 (Banach-Necas-Babuška). Let $\mathbf{X}$ be a Hilbert space, $\widetilde{\mathcal{A}}: \mathbf{X} \times \mathbf{X} \rightarrow$ $\mathbb{R}$ be a bilinear continuous form, and $\mathbf{F} \in \mathbf{X}^{\prime}$. Then problem (3.6) is well-posed (i.e., there exists a unique solution which depends continuously on the data) if and only if the following conditions are satisfied: 
(BNB1) There exists $\sigma>0$ such that $\inf _{\chi \in \mathbf{X}} \sup _{\bar{\chi} \in \mathbf{X}} \frac{\widetilde{\mathcal{A}}(\chi, \bar{\chi})}{\|\chi\|\|\bar{\chi}\|} \geq \sigma$.

(BNB2) If $\widetilde{\mathcal{A}}(\boldsymbol{\chi}, \overline{\boldsymbol{\chi}})=0$ for all $\boldsymbol{\chi} \in \mathbf{X}$, then $\overline{\boldsymbol{\chi}}=0$.

Moreover, the following a priori estimate holds:

$$
\|\chi\| \leq \frac{1}{\sigma}\|\mathbf{F}\|_{\mathbf{X}^{\prime}}
$$

Remark 5. If $\mathbf{A}: \mathbf{X} \rightarrow \mathbf{X}^{\prime}$ and $\mathbf{A}^{*}: \mathbf{X}^{\prime} \rightarrow \mathbf{X}$ are the continuous linear operators defined as $(\mathbf{A} \boldsymbol{\chi}, \overline{\boldsymbol{\chi}})=\widetilde{\mathcal{A}}(\boldsymbol{\chi}, \overline{\boldsymbol{\chi}})=\left(\boldsymbol{\chi}, \mathbf{A}^{*} \overline{\boldsymbol{\chi}}\right)$ for all $\boldsymbol{\chi}, \overline{\boldsymbol{\chi}} \in \mathbf{X}$, then the following equivalences hold:

(BNB1) $\Longleftrightarrow \quad \operatorname{ker} \mathbf{A}=\{0\}$ and Img $\mathbf{A}$ is closed $\Longleftrightarrow \mathbf{A}^{*}$ is surjective.

(BNB2) $\Longleftrightarrow \quad$ ker $\mathbf{A}^{*}=\{0\} \quad \Longleftrightarrow \quad \mathbf{A}^{*}$ is injective.

By using Theorem 3.1, an additional bound of $\partial_{z} p$ in $L^{2}(\Omega)$ can be proved.

TheOREM 3.2. There exists a unique solution of (3.2)-(3.4), (u, $v, p) \in \mathbf{U} \times V \times \widehat{P}$, which satisfies the a priori estimate

$$
\left\|\left(\nabla \mathbf{u}, \partial_{z} v, p, \partial_{z} p\right)\right\| \leq \frac{1}{\sigma}\|\mathbf{f}\|_{\mathbf{U}^{\prime}},
$$

where $\sigma \in(0,1 / 2]$ depens only on $\beta_{p}$ (the constant given in $\left.(I S)^{P}\right)$.

Proof. Problem (3.2)-(3.4) can be written as (3.6), where $\widetilde{\mathcal{A}}(\cdot, \cdot)$ is the bilinear form defined in (3.5). Then, thanks to Theorem 3.1, it suffices to verify that (BNB1) and (BNB2) hold.

It is easy to check (BNB2) because, given $\bar{\chi}=(\overline{\mathbf{u}}, \bar{v}, \bar{p}) \in \mathbf{X}$ such that $\widetilde{\mathcal{A}}(\boldsymbol{\chi}, \overline{\boldsymbol{\chi}})=0$ for all $\chi \in \mathbf{X}$, if we take $\chi=\bar{\chi}$, then

$$
\begin{aligned}
0=\widetilde{\mathcal{A}}(\overline{\boldsymbol{\chi}}, \overline{\boldsymbol{\chi}}) & =\nu\|\nabla \overline{\mathbf{u}}\|^{2}+\nu\left\|\partial_{z} \bar{v}\right\|^{2}+\left\|\partial_{z} \bar{p}\right\|^{2}+\nu\left(\nabla_{\mathbf{x}} \cdot \overline{\mathbf{u}}, \partial_{z} \bar{v}\right) \\
& \geq \frac{\nu}{2}\left(\|\nabla \overline{\mathbf{u}}\|^{2}+\left\|\partial_{z} \bar{v}\right\|^{2}\right)+\left\|\partial_{z} \bar{p}\right\|^{2}
\end{aligned}
$$

where (2.20) has been applied. Hence, $\overline{\mathbf{u}}=0, \bar{v}=0$, and $\partial_{z} \bar{p}=0$. But also $\bar{p}=0$ because, since $\overline{\mathbf{u}}, \bar{v}$, and $\partial_{z} \bar{p}$ vanish, $0=\widetilde{\mathcal{A}}(\chi, \overline{\boldsymbol{\chi}})=(\nabla \cdot(\mathbf{u}, v), \bar{p})$ for all $(\mathbf{u}, v) \in \mathbf{W}$, and hence the inf-sup condition $(I S)^{P}$ implies $\bar{p}=0$.

Let us also prove that (BNB1) holds or, equivalently, there exists $\sigma>0$ such that for each $\chi \in \mathbf{X}$, we can select $\bar{\chi}=\bar{\chi}(\chi) \in \mathbf{X}$ such that

$$
\widetilde{\mathcal{A}}(\chi, \bar{\chi}) \geq \sigma\|\chi\|^{2} \quad \text { and } \quad\|\bar{\chi}\| \leq\|\chi\| .
$$

Fix $\chi=(\mathbf{u}, v, p) \in \mathbf{X}$ :

Step 1. If we define $\bar{\chi}_{1}=\chi \in \mathbf{X}$ and take $\bar{\chi}=\bar{\chi}_{1}$ in (3.5), then

$$
\widetilde{\mathcal{A}}\left(\chi, \bar{\chi}_{1}\right) \geq \frac{\nu}{2}\left(\|\nabla \mathbf{u}\|^{2}+\left\|\partial_{z} v\right\|^{2}\right)+\left\|\partial_{z} p\right\|^{2} .
$$

Step 2. Fixing $p \in \widehat{P},(I S)^{P}$ condition is equivalent to the existence of $\mathbf{w}_{2}=$ $\left(\mathbf{u}_{2}, v_{2}\right) \in \mathbf{W}$ (depending on $p$ ) such that

$$
\left(\nabla \cdot\left(\mathbf{u}_{2}, v_{2}\right), p\right) \geq \beta_{p}\|p\|^{2} \text { and }\left\|\left(\nabla \mathbf{u}_{2}, \partial_{z} v_{2}\right)\right\| \leq\|p\| .
$$

Copyright (c) by SIAM. Unauthorized reproduction of this article is prohibited. 
Consequently, taking $\bar{\chi}_{2}=-\left(\mathbf{u}_{2}, v_{2}, 0\right) \in \mathbf{X}$,

$$
\begin{gathered}
\widetilde{\mathcal{A}}\left(\boldsymbol{\chi}, \overline{\boldsymbol{\chi}}_{2}\right)=-\nu\left(\left(\nabla \mathbf{u}, \nabla \mathbf{u}_{2}\right)-\left(\partial_{z} v, \partial_{z} v_{2}\right)-\left(\nabla_{\mathbf{x}} \cdot \mathbf{u}, \partial_{z} v_{2}\right)\right)+\left(p, \nabla \cdot\left(\mathbf{u}_{2}, v_{2}\right)\right) \\
\geq-\nu\left(\frac{\nu}{\beta_{p}}\|\nabla \mathbf{u}\|^{2}+\frac{\beta_{p}}{4 \nu}\|p\|^{2}+\frac{\nu}{\beta_{p}}\left\|\partial_{z} v\right\|^{2}\right. \\
\left.\quad+\frac{\beta_{p}}{4 \nu}\|p\|^{2}+\frac{\nu}{\beta_{p}}\|\nabla \mathbf{u}\|^{2}+\frac{\beta_{p}}{4 \nu}\|p\|^{2}\right)+\beta_{p}\|p\|^{2} \\
=-\frac{2 \nu^{2}}{\beta_{p}}\|\nabla \mathbf{u}\|^{2}-\frac{\nu^{2}}{\beta_{p}}\left\|\partial_{z} v\right\|^{2}+\frac{\beta_{p}}{4}\|p\|^{2} .
\end{gathered}
$$

Step 3. Finally, if we define $\bar{\chi}=\frac{1}{2} \bar{\chi}_{1}+\frac{1}{K} \bar{\chi}_{2}$ with $K \geq 2$ big enough, then $\|\bar{\chi}\| \leq\|\chi\|$, because $\left\|\bar{\chi}_{1}\right\|=\|\chi\|$ and $\left\|\bar{\chi}_{2}\right\|=\left\|\nabla \mathbf{u}_{2}, \partial_{z} v_{2}\right\| \leq\|p\| \leq\|\chi\|$. Hence,

$$
\widetilde{\mathcal{A}}(\chi, \bar{\chi}) \geq\left(\frac{\nu}{4}-\frac{2 \nu^{2}}{K \beta_{p}}\right)\|\nabla \mathbf{u}\|^{2}+\left(\frac{\nu}{4}-\frac{\nu^{2}}{K \beta_{p}}\right)\left\|\partial_{z} v\right\|^{2}+\frac{\beta_{p}}{4 K}\|p\|^{2}+\frac{1}{2}\left\|\partial_{z} p\right\|^{2} .
$$

Moreover, $K$ can be chosen such that latter constants are positive. For example, if $K=2+8 \nu / \beta_{p}=\left(2 \beta_{p}+8 \nu\right) / \beta_{p}$, then the previous inequality reads

$$
\widetilde{\mathcal{A}}(\chi, \bar{\chi}) \geq \frac{\beta_{p}}{4 \beta_{p}+16 \nu}\|\nabla \mathbf{u}\|^{2}+\frac{\beta_{p}+2 \nu^{2}}{4 \beta_{p}+16 \nu}\left\|\partial_{z} v\right\|^{2}+\frac{\beta_{p}^{2}}{8 \beta_{p}+32 \nu}\|p\|^{2}+\frac{1}{2}\left\|\partial_{z} p\right\|^{2},
$$

and hence (3.9) holds for $\sigma=\min \left\{\beta_{p} /\left(4 \beta_{p}+16 \nu\right), \beta_{p}^{2} /\left(8 \beta_{p}+32 \nu\right), 1 / 2\right\}$.

3.2. Well-posedness of the discrete scheme and error estimates. Let $\mathbf{U}_{h}, V_{h}, \widehat{P}_{h}$ be three conforming FE spaces contained in $\mathbf{U}, V, \widehat{P}$, respectively, and let us consider the following finite-dimensional problem: find $\mathbf{u}_{h} \in \mathbf{U}_{h}, v_{h} \in V_{h}$, and $p_{h} \in \widehat{P}_{h}$ such that

$$
\begin{aligned}
\nu\left(\nabla \mathbf{u}_{h}, \nabla \overline{\mathbf{u}}_{h}\right)-\left(p_{h}, \nabla_{\mathbf{x}} \cdot \overline{\mathbf{u}}_{h}\right) & =\left(\mathbf{f}, \overline{\mathbf{u}}_{h}\right) & & \forall \overline{\mathbf{u}}_{h} \in \mathbf{U}_{h}, \\
\nu\left(\nabla \cdot\left(\mathbf{u}_{h}, v_{h}\right), \partial_{z} \bar{v}_{h}\right)-\left(p_{h}, \partial_{z} \bar{v}_{h}\right) & =0 & & \forall \bar{v}_{h} \in V_{h}, \\
\left(\nabla \cdot\left(\mathbf{u}_{h}, v_{h}\right), \bar{p}_{h}\right)+\left(\partial_{z} p_{h}, \partial_{z} \bar{p}_{h}\right) & =0 & & \forall \bar{p}_{h} \in \widehat{P}_{h} .
\end{aligned}
$$

The argument in the previous subsection can be extended to this Galerkin conforming approximation. In this case, Theorem 3.1 is simplified due to the fact of $\mathbf{X}_{h} \subset \mathbf{X}$ is a finite-dimensional space. Indeed, scheme (3.11)-(3.13) can be reformulated as

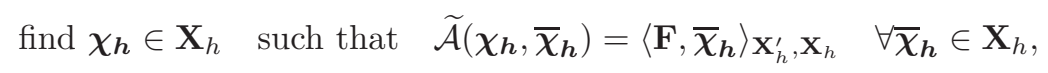

where $\left\langle\mathbf{F}, \bar{\chi}_{\boldsymbol{h}}\right\rangle_{\mathbf{X}_{h}^{\prime}, \mathbf{X}_{h}}=\left\langle\mathbf{f}, \overline{\mathbf{u}}_{h}\right\rangle_{\mathbf{U}_{h}^{\prime}, \mathbf{U}_{h}}$, and hence (3.14) is equivalent to a square linear system of matrix $A_{h}$ and the discrete versions of (BNB1) and (BNB2) are equivalents (see, e.g., [11, Proposition 2.21]), that is,

$$
(\mathrm{BNB} 1)_{h} \Leftrightarrow \operatorname{ker} A_{h}=\{0\} \Leftrightarrow \operatorname{rank} A_{h}=\operatorname{dim} \mathbf{X}_{h} \Leftrightarrow(\mathrm{BNB} 2)_{h} .
$$

Therefore, the following result can be stated.

THEOREM 3.3. If $\left(\mathbf{U}_{h}, V_{h}\right)-\widehat{P}_{h}$ satisfies $(I S)_{h}^{P}$ with constant $\gamma_{p}$ (independent of $h)$, there exists a unique solution $\left(\mathbf{u}_{h}, v_{h}, p_{h}\right) \in \mathbf{U}_{h} \times V_{h} \times \widehat{P}_{h}$ of (3.11)-(3.13), which satisfies the following a priori estimates:

$$
\left\|\left(\nabla \mathbf{u}_{h}, \partial_{z} v_{h}, p_{h}, \partial_{z} p_{h}\right)\right\| \leq \frac{1}{\tau}\|\mathbf{f}\|_{\mathbf{U}^{\prime}}
$$

Copyright $@$ by SIAM. Unauthorized reproduction of this article is prohibited. 
where $\tau \in(0,1 / 2]$ is a constant independent of $h$. (In fact, $\tau$ only depends on $\nu$ and $\gamma_{p}$; the latter is the constant in $(I S)_{h}^{P}$.)

Proof. Let us consider problem (3.14) for $\mathbf{X}_{h}=\mathbf{U}_{h} \times V_{h} \times \widehat{P}_{h}$. In the finitedimensional case, the following condition is sufficient for the well-posedness of scheme (3.14) (see, e.g., [11, Theorem 2.6]):

$(\mathrm{BNB} 1)_{h} \quad$ There exists $\tau>0$ such that $\inf _{\boldsymbol{\chi} \in \mathbf{X}_{h}} \sup _{\overline{\boldsymbol{\chi}} \in \mathbf{X}_{h}} \frac{\widetilde{\mathcal{A}}(\boldsymbol{\chi}, \overline{\boldsymbol{\chi}})}{\|\chi\|\|\bar{\chi}\|} \geq \tau$.

Moreover, in this case, the following a priori estimate holds (coinciding with estimate (3.15)):

$$
\left\|\chi_{h}\right\| \leq \frac{1}{\tau}\|\mathbf{F}\|_{\mathbf{X}^{\prime}}
$$

Finally, assuming $(I S)_{h}^{P}$ with constant $\gamma_{p}$ independent of $h,(\mathrm{BNB} 1)_{h}$ can be satisfied as in Theorem 3.2 with $\tau=\min \left\{\gamma_{p} /\left(4 \gamma_{p}+16 \nu\right), \gamma_{p}^{2} /\left(8 \gamma_{p}+32 \nu\right), 1 / 2\right\}$, which is independent of $h$.

Once the well-posedness of the scheme (3.11)-(3.13) is proved, the following error estimates, including the $L^{2}(\Omega)$-norm in $\partial_{z} p_{h}$, can be obtained.

TheOREm 3.4. Let $(\mathbf{u}, v, p)$ and $\left(\mathbf{u}_{h}, v_{h}, p_{h}\right)$ be the solution of problems (3.2)(3.4) and (3.11)-(3.13), respectively. Assume that there is $\gamma_{p}>0$ (independent of $h$ ) satisfying $(I S)_{h}^{P}$. Then there is a constant $C>0$ depending on $\gamma_{p}$ (and independent on $h$ ) such that

$$
\begin{aligned}
& \left\|\left(\nabla\left(\mathbf{u}-\mathbf{u}_{h}\right), \partial_{z}\left(v-v_{h}\right),\left(p-p_{h}\right), \partial_{z}\left(p-p_{h}\right)\right)\right\| \\
& \quad \leq C\left(\inf _{\overline{\mathbf{u}}_{h} \in \mathbf{U}_{h}}\left\|\nabla\left(\mathbf{u}-\overline{\mathbf{u}}_{h}\right)\right\|+\inf _{\bar{v}_{h} \in V_{h}}\left\|\partial_{z}\left(v-\bar{v}_{h}\right)\right\|+\inf _{\bar{p}_{h} \in \widehat{P}_{h}}\left\|p-\bar{p}_{h}\right\|_{H_{z}^{1}}\right) .
\end{aligned}
$$

Proof. Under the assumptions above, $\boldsymbol{\chi}=(\mathbf{u}, v, p)$ and $\chi_{h}=\left(\mathbf{u}_{h}, v_{h}, p_{h}\right)$ are the solution of (3.6) and (3.14), respectively. It suffices to apply the well-known generalization of Cea's lemma for generalized elliptic problems (see, e.g., [12]), which reads (using $\|a\| \leq 2 \nu$ ):

$$
\left\|\chi-\chi_{\boldsymbol{h}}\right\|_{\mathbf{X}} \leq\left(1+\frac{2 \nu}{\gamma_{p}}\right) \inf _{\bar{\chi}_{h} \in \mathbf{X}_{h}}\left\|\chi-\bar{\chi}_{\boldsymbol{h}}\right\|_{\mathbf{X}} .
$$

Then the definition of $\|\cdot\|_{\mathbf{X}}$ implies estimate (3.16).

Remark 6 (convergence orders). Order $O(h)$ is obtained, even for $\partial_{z} p$ in $L^{2}(\Omega)$, for the combination $\mathcal{P}_{1, b}-\mathcal{P}_{1}$ if $\mathbf{u}, v, p \in H^{2}(\Omega)$, which improves the non- $\partial_{z} p$-regularized scheme (see Remark 3). But now, order $O\left(h^{2}\right)$ cannot be reached for $\mathcal{P}_{2}-\mathcal{P}_{1}$ in generic meshes (because a best approximation, for example $\mathcal{P}_{2}$, would be required for pressure).

Our numerical simulations (see Test 4 ) confirm this fact for $\mathcal{P}_{1, b}-\mathcal{P}_{1}$. About $\mathcal{P}_{2}-\mathcal{P}_{1}$, Test 4 suggests that, in the case of structured meshes with constant depth, order $O\left(h^{2}\right)$ is obtained in energy norms. This fact can be justified as follows: if $\mathcal{T}_{h}$ is furnished as a tensor product of a surface mesh, $\mathcal{T}_{h}^{S}$, times a set of depth layers, defined by a partition $-D=z_{0}<z_{1}<\cdots<z_{n}=0$, then one can define

$$
Q_{h}^{S}=\left\{q_{h} \in L_{0}^{2}(S) \cap C^{0}(\bar{S}) /\left.q_{h}\right|_{T} \in \mathcal{P}_{1} \quad \forall T \in \mathcal{T}_{h}^{S}\right\} .
$$

Taking into account $\partial_{z} p=0$ and the fact that, in this kind of meshes, any $\bar{p}_{h} \in P_{h}$ (with $\mathcal{P}_{1}$ approximation) such that $\partial_{z} \bar{p}_{h}=0$ can be identified with some $\bar{q}_{h} \in Q_{h}^{S}$ (in 
fact, $\left.\bar{q}_{h}(x)=\bar{p}_{h}(x, z)\right)$ and $\left\|p-\bar{p}_{h}\right\|_{H_{z}^{1}}=\left\|p-\bar{p}_{h}\right\|$, then

$$
\inf _{\bar{p}_{h} \in P_{h}}\left\|p-\bar{p}_{h}\right\|_{H_{z}^{1}} \leq \inf _{\substack{\bar{p}_{h} \in P_{h} \\ \partial_{z} \bar{p}_{h}=0}}\left\|p-\bar{p}_{h}\right\|=\sqrt{D} \inf _{\bar{q}_{h} \in Q_{h}^{S}}\left\|p-\bar{q}_{h}\right\|_{L^{2}(S)}=O\left(h^{2}\right) .
$$

3.3. The problem without stabilization of vertical velocity. Let us consider now the following variational system: find $(\mathbf{u}, v) \in \mathbf{W}=\mathbf{U} \times V=\mathbf{H}_{b, l}^{1}(\Omega) \times$ $H_{z, 0}^{1}(\Omega)$ and $p \in \widehat{P}$ (space defined in (3.1)) such that

$$
\begin{aligned}
\nu(\nabla \mathbf{u}, \nabla \overline{\mathbf{u}})-\left(p, \nabla_{\mathbf{x}} \cdot \overline{\mathbf{u}}\right) & =(\mathbf{f}, \overline{\mathbf{u}}) & & \forall \overline{\mathbf{u}} \in \mathbf{U}, \\
\left(p, \partial_{z} \bar{v}\right) & =0 & & \forall \bar{v} \in V, \\
(\nabla \cdot(\mathbf{u}, v), \bar{p})+\left(\partial_{z} p, \partial_{z} \bar{p}\right) & =0 & & \forall \bar{p} \in \widehat{P} .
\end{aligned}
$$

This problem is obtained by adding, directly to the hydrostatic Stokes equations (1.14)(1.16), the consistent term $\left(\partial_{z} p, \partial_{z} \bar{p}\right)$.

Once again, since $\partial_{z} V \not \subset \widehat{P}$, it is not straightforward to guarantee the equivalence of problems (3.17)-(3.19) and (3.2)-(3.4) or (1.14)-(1.16). It is also clear that the solution of (1.14)-(1.16) satisfies (3.17)-(3.19) if $p \in \widehat{P}$. But, in this case, the stabilization of $v$ which was introduced in section 2 is broken and, even although we would rely on the stability condition $(I S)^{V}$, the argument employed in sections 3.1 and 3.2 (based on Banach-Necas-Babuška Theorem 3.1) does not lead to the well-posedness of problem (3.17)-(3.19). As we will see, the key is that estimates for $v$ in $H_{z}^{1}(\Omega)$ cannot be now obtained, even using $(I S)^{V}$.

To delve into this question, let us write (3.17)-(3.19) as follows: find $\chi \in \mathbf{X}$ such that

$$
\widetilde{\mathcal{A}}(\chi, \bar{\chi})=\langle\mathbf{F}, \bar{\chi}\rangle_{\mathbf{X}^{\prime}, \mathbf{X}} \quad \forall \bar{\chi} \in \mathbf{X}
$$

where

$$
\widetilde{\mathcal{A}}(\boldsymbol{\chi}, \overline{\boldsymbol{\chi}}):=\nu(\nabla \mathbf{u}, \nabla \overline{\mathbf{u}})+\left(\partial_{z} p, \partial_{z} \bar{p}\right)-(p, \nabla \cdot(\overline{\mathbf{u}}, \bar{v}))+(\nabla \cdot(\mathbf{u}, v), \bar{p})
$$

for each $\chi=(\mathbf{w}, p), \bar{\chi}=(\overline{\mathbf{w}}, \bar{p}) \in \mathbf{X}$ with $\mathbf{w}=(\mathbf{u}, v)$ and $\overline{\mathbf{w}}=(\overline{\mathbf{u}}, \bar{v})$, where $\langle\mathbf{F}, \bar{\chi}\rangle_{\mathbf{X}^{\prime}, \mathbf{X}}$ is defined in (3.7). In this context, we try to use Theorem 3.1, as in the proof of Theorem 3.2. For showing (BNB1), fix $\chi=(\mathbf{u}, v, p) \in \mathbf{X}$ : $\left\|\partial_{z} p\right\|^{2}$.

Step 1 . Take $\bar{\chi}_{1}=\chi \in \mathbf{X}$ and $\bar{\chi}=\bar{\chi}_{1}$ in (3.21), and then $\widetilde{\mathcal{A}}\left(\boldsymbol{\chi}, \bar{\chi}_{1}\right)=\nu\|\nabla \mathbf{u}\|^{2}+$

Step 2. As in proof of Theorem 3.2, we can extract some estimate for $\|p\|$, using $(I S)^{P}$ as follows: fix $p \in \widehat{P}$, and there exists $\mathbf{w}_{2}=\left(\mathbf{u}_{2}, v_{2}\right) \in \mathbf{W}$ (depending on $p$ ) such that (3.10) holds. Taking $\bar{\chi}_{2}=-\left(\mathbf{u}_{2}, v_{2}, 0\right) \in \mathbf{X}$ and using (3.10), one has

$$
\begin{aligned}
\widetilde{\mathcal{A}}\left(\boldsymbol{\chi}, \bar{\chi}_{2}\right) & =-\nu\left(\nabla \mathbf{u}, \nabla \mathbf{u}_{2}\right)+\left(p, \nabla \cdot\left(\mathbf{u}_{2}, v_{2}\right)\right) \\
& \geq-\nu\left(\frac{\nu}{2 \beta_{p}}\|\nabla \mathbf{u}\|^{2}+\frac{\beta_{p}}{2 \nu}\|p\|^{2}\right)+\beta_{p}\|p\|^{2} \geq-\frac{\nu^{2}}{2 \beta_{p}}\|\nabla \mathbf{u}\|^{2}+\frac{\beta_{p}}{2}\|p\|^{2} .
\end{aligned}
$$

Step 3. Finally, we must obtain any estimate for $\left\|\partial_{z} v\right\|$, which does not appear as a coercive term in Step 1. If we apply $(I S)^{V}$, there exists $\widetilde{p} \in P$ (depending on $v$ ) such that

$$
\left(\partial_{z} v, \widetilde{p}\right) \geq \beta_{v}\left\|\partial_{z} v\right\|^{2} \quad \text { and } \quad\|\widetilde{p}\| \leq\left\|\partial_{z} v\right\|
$$

Copyright $@$ by SIAM. Unauthorized reproduction of this article is prohibited. 
Taking $\bar{\chi}_{3}=-(0,0, \widetilde{p}) \in \mathbf{X}$ and using (3.22),

$$
\begin{aligned}
\widetilde{\mathcal{A}}\left(\boldsymbol{\chi}, \overline{\boldsymbol{\chi}}_{3}\right) & =\left(\partial_{z} p, \partial_{z} \widetilde{p}\right)+\left(\nabla_{\mathbf{x}} \mathbf{u}, \widetilde{p}\right)+\left(\partial_{z} v, \widetilde{p}\right) \\
& \geq-\frac{1}{2}\left\|\partial_{z} p\right\|^{2}-\frac{1}{2}\left\|\partial_{z} \widetilde{p}\right\|^{2}-\frac{1}{2 \beta_{v}}\|\nabla \mathbf{u}\|^{2}+\frac{\beta_{v}}{2}\left\|\partial_{z} v\right\|^{2} .
\end{aligned}
$$

At this point, the stability of (3.17)-(3.19) cannot be shown (at least using this reasoning) because a term which is not bounded, $\left\|\partial_{z} \widetilde{p}\right\|$, appears even for FE spaces satisfying $(I S)^{V}$. This suggests the instability of formulation (3.17)-(3.19) (although it is not proved) due to the fact that $\left\|\partial_{z} v\right\|$ is not controlled by coercivity. Our computer experiments support this conjecture (see Test 2).

Remark 7. To bound $\left\|\partial_{z} v\right\|$ in Step 3, the following variant of $(I S)^{V}$ would be sufficient:

$$
\sup _{0 \neq \bar{p} \in \widehat{P}} \frac{\left(\partial_{z} v, \bar{p}\right)}{\|\bar{p}\|_{H_{z}^{1}}} \geq \beta_{v}\left\|\partial_{z} v\right\| \quad \forall v \in V .
$$

The problem is that this condition involves a stronger norm for $\bar{p}$ and, in this sense, its verification is not straightforward.

4. Numerical simulations. The first numerical test shows, in practice, some of the advantages of the schemes presented in this paper. Specifically, flexibility for the approximation of different domains by two-dimensional (2D) or 3D meshes, using Stokes-stable FE, like Taylor-Hood $\mathcal{P}_{2}-\mathcal{P}_{1}$ or minielement $\mathcal{P}_{1, b}-\mathcal{P}_{1}$ and mesh adaptivity without additional difficulties. Latter tests are centered in validation of theoretical results and the numerical approach of the convergence rates.

TEST 1 (Gibraltar Strait 2D cavity test). In order to show the flexibility of schemes (2.4)-(2.6) and (3.11)-(3.13) for handling arbitrary domains for any (even discontinuous) depth function, with or without talus under $\partial S$, we have considered a $2 D$ cavity test in a domain defined by real data.

More specifically, the $2 D$ domain has been built as follows: first, we consider (in Cartesian coordinates) the segment, $S$, joining two points defined by respective latitude $35.02^{\circ}, 37^{\circ}$ and longitude $-11^{\circ}, 2^{\circ}$, which cross the Gibraltar Strait (see Figure $2(\mathrm{a}))$. Then we define the domain $\Omega$ using a depth function which is constructed using bathymetry data (from U.S.A. NOAA, [24]). Then, we build the bathymetry graphic showed in Figure 2(b) and manipulate it, filling the $2 D$ domain with black color. Then a program (in this test, we have used the FE language FreeFem $++[20]$ ) can transform this image into an unstructured mesh, and our simulation can be performed. Figure 2(b) shows the bottom profile, where well known geographic landforms can be observed, namely, the Spartel and Camarinal sills near longitude $-6^{\circ}$.

Exploiting the fact that our numerical scheme allows us to take advantage of advanced FE techniques, such as mesh adaptivity, it has been easy to use FreeFem++ to refine the original mesh, performing one step of mesh adaptivity where the Hessian of the following function was used as indicator:

$$
u_{h} /\left\|u_{h}\right\|_{\infty}+v_{h} /\left\|v_{h}\right\|_{\infty}+p_{h} /\left\|p_{h}\right\|_{\infty} .
$$

Figures 2(c) and 2(d) show, respectively, the velocity streamlines and pressure isolines obtained with $\mathcal{P}_{2}-\mathcal{P}_{1} F E$ for the $v$-stabilized and $\partial_{z}$-regularized scheme (although the non- $\partial_{z}$ p-regularized scheme presents similar qualitative behavior). Dirichlet boundary conditions $\mathbf{u}=1, v=0$ on the surface and $\mathbf{u}=v=0$ on the bottom have been imposed, and one step of the mesh adapting technique has been used.

Copyright (C) by SIAM. Unauthorized reproduction of this article is prohibited. 


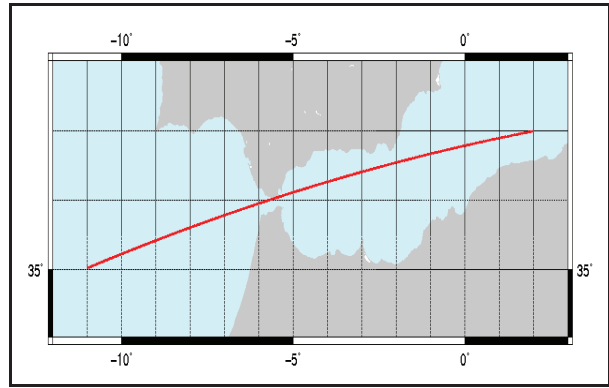

(a) Surface domain

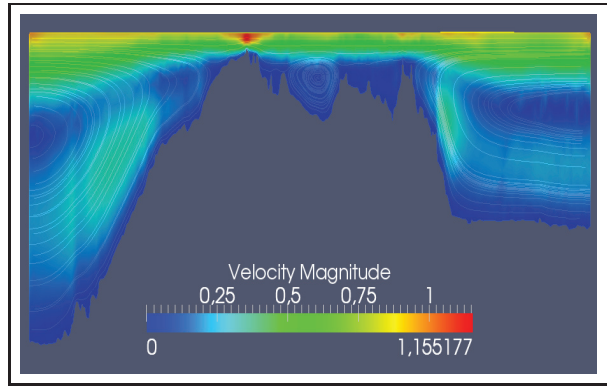

(c) Velocity streamlines

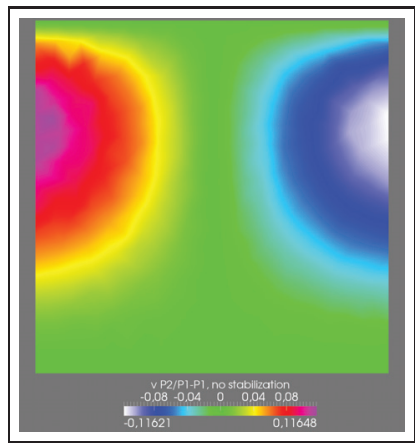

(e) $\left(\mathcal{P}_{2}, \mathcal{P}_{1}\right)-\mathcal{P}_{1}$

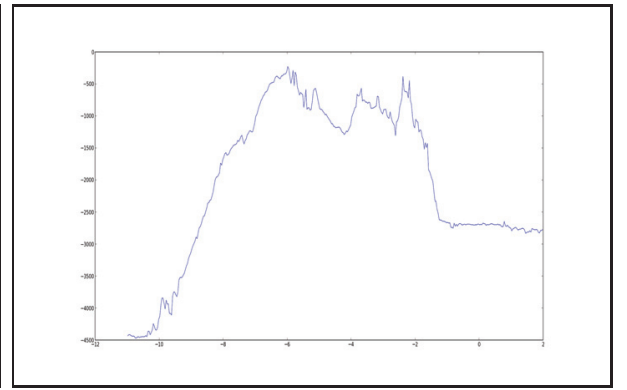

(b) Bathymetry

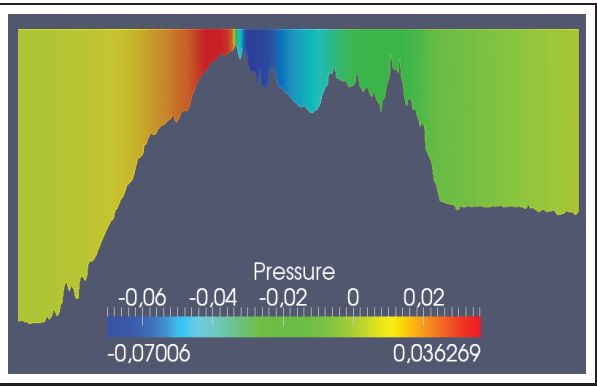

(d) Pressure contour

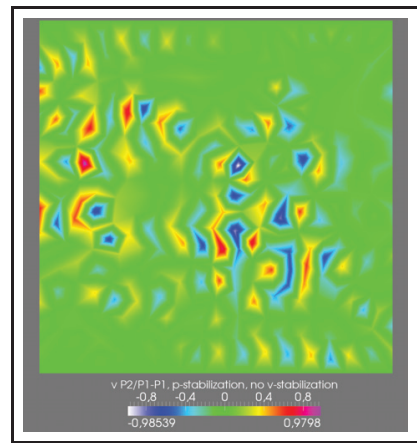

(f) $\left(\mathcal{P}_{2}, \mathcal{P}_{1}\right)-\mathcal{P}_{1}$ and $p$-regularized.

FIG. 2. $v$ graphics: left for the non-v-stabilized and non-p-regularized scheme; right for the non-v-stabilized and $p$-regularized scheme.

Resulting Figures 2(c) and 2(d) are according the expected qualitative behavior: velocity recirculation (note that no Dirichlet condition is imposed on sidewalls) and hydrostatic pressure.

TEST 2 (study of the $p$-regularized but not $v$-stabilized scheme). As was shown in section 3.3, formulation (3.17)-(3.19) may be unstable due to the fact that $\|v\|_{L^{2}}$ cannot be controlled, even if $(I S)^{V}$ holds. A cavity test in the square $\Omega=(0,1) \times(-1,0)$ supports this conjecture. Specifically, Figure $2(\mathrm{f})$ shows some spurious oscillations for the vertical velocity when $\left(\mathcal{P}_{2}, \mathcal{P}_{1}\right)-\mathcal{P}_{1}$ is applied to scheme (3.17)-(3.19). See that the combination $\left(\mathcal{P}_{2}, \mathcal{P}_{1}\right)-\mathcal{P}_{1}$ in unstructured meshes could satisfy $(I S)_{h}^{V}$ and also satisfy $(I S)_{h}^{P}$. In fact, if the non-v-stabilized and non- $\partial_{z} p$-regularized scheme is used, oscillations disappear (Figure 2(e)). 


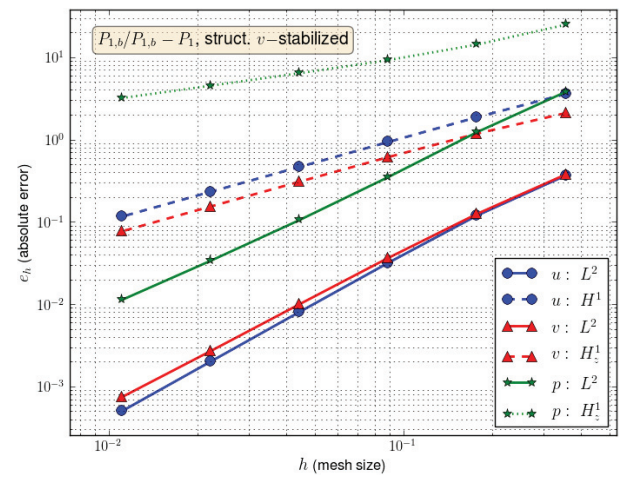

(a) Errors for $\mathcal{P}_{1, b}-\mathcal{P}_{1}$

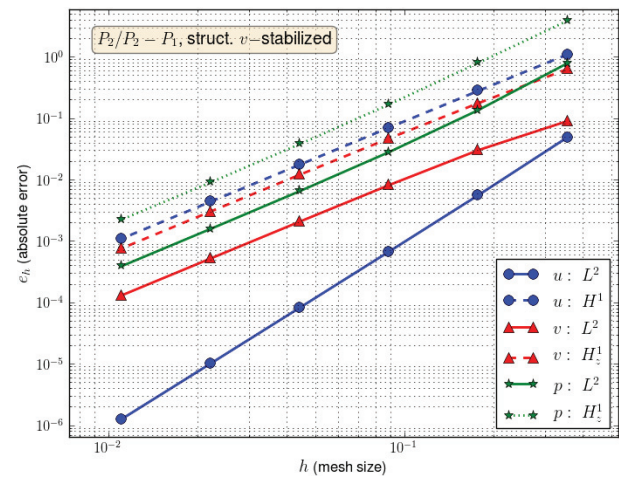

(b) Errors for $\mathcal{P}_{2}-\mathcal{P}_{1}$

FIG. 3. Velocity and pressure errors for $\mathcal{P}_{1, b}-\mathcal{P}_{1}$ and $\mathcal{P}_{2}-\mathcal{P}_{1}$ (v-stabilized scheme).

TABLE 1

Error orders for $\mathcal{P}_{1, b}-\mathcal{P}_{1}$ and $\mathcal{P}_{2}-\mathcal{P}_{1}$ in a structured mesh (v-stabilized scheme).

\begin{tabular}{|c|c|c|c|c|c|c|c|c|c|c|c|}
\hline$h$ & & $2^{-3}$ & $2^{-4}$ & $2^{-5}$ & $2^{-6}$ & $2^{-7}$ & $2^{-3}$ & $2^{-4}$ & $2^{-5}$ & $2^{-6}$ & $2^{-7}$ \\
\hline & & \multicolumn{5}{|c|}{$\mathcal{P}_{1, b}-\mathcal{P}_{1}$} & \multicolumn{5}{|c|}{$\mathcal{P}_{2}-\mathcal{P}_{1}$} \\
\hline \multirow{2}{*}{$\mathbf{u}$} & $L^{2}$ & 1.616 & 1.908 & 1.982 & 1.999 & 2.002 & 3.129 & 3.047 & 3.029 & 3.018 & 3.010 \\
\hline & $H_{0}^{1}$ & 0.936 & 1.001 & 1.004 & 1.002 & 1.001 & 1.967 & 1.987 & 1.999 & 2.001 & 2.001 \\
\hline \multirow[t]{2}{*}{$v$} & $L^{2}$ & 1.591 & 1.775 & 1.867 & 1.888 & 1.857 & 1.572 & 1.887 & 1.972 & 1.993 & 1.998 \\
\hline & $H_{z, 0}^{1}$ & 0.830 & 0.947 & 0.992 & 1.000 & 1.001 & 1.846 & 1.899 & 1.964 & 1.989 & 1.997 \\
\hline \multirow{2}{*}{$p$} & $L^{2}$ & 1.62 & 1.812 & 1.7 & 1.6 & 1.579 & 2.532 & 2.267 & 2.109 & 2.042 & 2.017 \\
\hline & $H_{z, 0}^{1}$ & 0.801 & 0.626 & 0.528 & 0.502 & 0.500 & 2.278 & 2.245 & 2.140 & 2.070 & 2.015 \\
\hline
\end{tabular}

TEST 3 (error orders for the $v$-stabilized scheme). With the aim of testing numerically error orders for the v-stabilized scheme (2.4)-(2.6), we have considered the unit square domain in $\mathbb{R}^{2}$ and the following exact solution of the hydrostatic problem (1.14)-(1.16):

$$
\begin{aligned}
& \mathbf{u}(x, y)=\cos (2 \pi x) \sin (2 \pi y)-\sin (2 \pi y), \quad v(x, y)=-\mathbf{u}(y, x), \\
& p(x, y)=2 \pi \cos (2 \pi x) .
\end{aligned}
$$

This solution was approximated using both minielement $\mathcal{P}_{1, b}-\mathcal{P}_{1}$ and Taylor-Hood $\mathcal{P}_{2}-\mathcal{P}_{1} F E$, and the absolute error was computed for different mesh sizes and norms. Figure 3 shows the resulting graphics, where absolute errors are plotted (in logarithmic scales).

Table 1 shows the numerical error orders (estimated as $\log \left(e_{h_{2}} / e_{h_{1}}\right) / \log \left(h_{2} / h_{1}\right)$ where $e_{h_{i}}$ are the absolute errors and $h_{1}<h_{2}$ travel through the mesh sizes $2^{-2}$, $\left.2^{-3}, \ldots, 2^{-8}\right)$. The results agree with Remark 3 :

- For $\mathcal{P}_{1, b}-\mathcal{P}_{1} F E$, optimal order $O(h)$ is suggested by the slope of segments in Figure 3(a) and values in Table 1 (left), in the "coercive" norms $H_{0}^{1}$ by $\mathbf{u}$, $H_{z, 0}^{1}$ by $v$, and $L^{2}$ by $p$. Also, $O\left(h^{2}\right)$ order in $L^{2}$ is suggested for $\mathbf{u}$. Note that these results are very similar to the ones arising for classical Stokes equations.

- For $\mathcal{P}_{2}-\mathcal{P}_{1}$, Figure 3(b) and Table 1 (right) also suggest optimal $O\left(h^{2}\right)$ order for $(\mathbf{u}, v, p)$ in the coercive norms. In $L^{2}$-norm, optimal $O\left(h^{3}\right)$ order is nu-

Copyright (C) by SIAM. Unauthorized reproduction of this article is prohibited. 


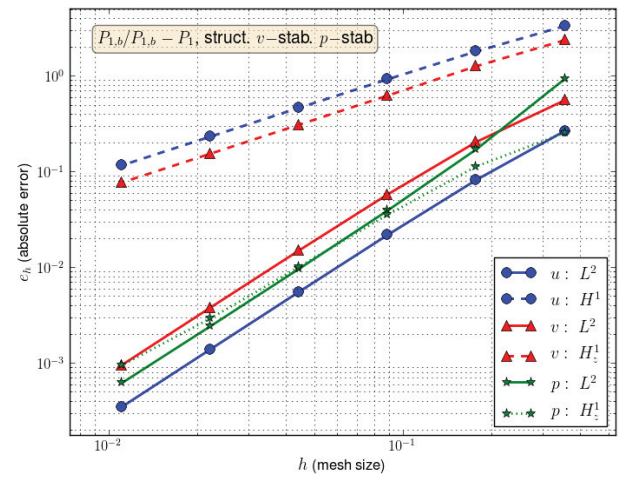

(a) Errors for $\mathcal{P}_{1, b}-\mathcal{P}_{1}$

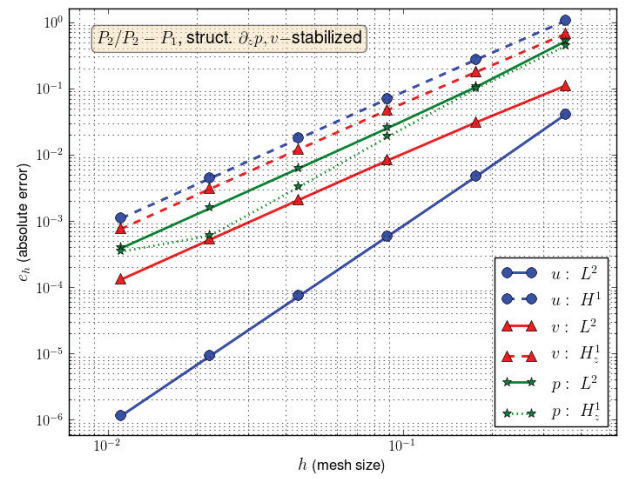

(b) Errors for $\mathcal{P}_{2}-\mathcal{P}_{1}$

FIG. 4. Velocity and pressure errors for $\mathcal{P}_{1, b}-\mathcal{P}_{1}$ and $\mathcal{P}_{2}-\mathcal{P}_{1}((p+v)$-stabilized scheme $)$.

TABLE 2

Error orders for $\mathcal{P}_{1, b}-\mathcal{P}_{1}$ and $\mathcal{P}_{2}-\mathcal{P}_{1}$ in a structured mesh $(p+v$-stabilized scheme $)$.

\begin{tabular}{|c|c|c|c|c|c|c|c|c|c|c|c|}
\hline \multirow[t]{2}{*}{$\bar{h}$} & & $2^{-3}$ & $2^{-4}$ & $2^{-5}$ & $2^{-6}$ & $2^{-7}$ & $2^{-3}$ & $2^{-4}$ & $2^{-5}$ & $2^{-6}$ & $2^{-7}$ \\
\hline & & \multicolumn{5}{|c|}{$\mathcal{P}_{1, b}-\mathcal{P}_{1}$} & \multicolumn{5}{|c|}{$\mathcal{P}_{2}-\mathcal{P}_{1}$} \\
\hline \multirow{2}{*}{$\mathbf{u}$} & $L^{2}$ & 1.701 & 1.908 & 1.975 & 1.994 & 1.999 & 3.092 & 3.014 & 3.001 & 3.000 & 3.000 \\
\hline & $H_{0}^{1}$ & 0.884 & 0.966 & 0.991 & 0.998 & 1.000 & 1.936 & 1.975 & 1.993 & 1.998 & 2.000 \\
\hline \multirow{2}{*}{$v$} & $L^{2}$ & 1.449 & 1.820 & 1.945 & 1.982 & 1.994 & 1.830 & 1.915 & 1.978 & 1.994 & 1.999 \\
\hline & $H_{z, 0}^{1}$ & 0.906 & 1.019 & 1.012 & 1.004 & 1.001 & 1.900 & 1.915 & 1.968 & 1.990 & 1.997 \\
\hline \multirow{2}{*}{$p$} & $L^{2}$ & 2.445 & 2.122 & 2.024 & 1.992 & 1.972 & 2.280 & 2.073 & 2.018 & 2.004 & 2.001 \\
\hline & $\overline{H_{z, 0}^{1}}$ & 1.171 & 1.676 & 1.809 & 1.788 & 1.629 & 2.108 & 2.437 & 2.519 & 2.440 & 0.792 \\
\hline
\end{tabular}

merically obtained for $\mathbf{u}$ but not for $v$. The case of $p$ in $H_{z}^{1}$, where $O\left(h^{2}\right)$ is clearly suggested (more than our numerical analysis predicts), is interesting. These simulations have been carried out in structured meshes, but the results in unstructured meshes are similar.

TEST 4 (error orders for the $(p+v)$-stabilized scheme). Finally, we show numerical error orders obtained for the $v$-stabilized and $\partial_{z} p$-regularized scheme (3.11)-(3.13). Exact solution (4.1)-(4.2) was again used. Both the minielement $\mathcal{P}_{1, b}-\mathcal{P}_{1}$ (Figure 4(a) and Table 2, left) and Taylor-Hood $\mathcal{P}_{2}-\mathcal{P}_{1}$ (Figure 4(b) and Table 2, right) have been considered, in structured meshes. The results agree with Remark 6:

- For $\mathcal{P}_{1, b}-\mathcal{P}_{1}$ FE (Figure 4(a) and Table 2, left), optimal order $O(h)$ is suggested in coercive norms for $(\mathbf{u}, v, p)$ and order $O\left(h^{2}\right)$ in $L^{2}$ for the velocity. These results agree with Theorem 3.4 and Remark 6 and coincide with Test 3 and classical Stokes error orders.

- For $\mathcal{P}_{2}-\mathcal{P}_{1} F E$ (Figure 4(b) and Table 2, right), optimal order $O\left(h^{2}\right)$ is obtained in coercive norms for $(\mathbf{u}, v, p)$, which is more than the theoretical results in section 3.2 predicted. Order $O\left(h^{3}\right)$ in $L^{2}$ is obtained again for $\mathbf{u}$, but only $O\left(h^{2}\right)$ for $v$. Also, $O\left(h^{2}\right)$ is initially suggested for $p$ in $H_{z}^{1}$, although our simulations do not reach even $O(h)$ for the smaller mesh sizes.

Comparing with Test 3 , error order is not improved by the $\partial_{z} p$-regularized scheme with $\mathcal{P}_{2}-\mathcal{P}_{1}$ FE. But with $\left(\mathcal{P}_{1}+\right.$ bubble $)-\mathcal{P}_{1}$, error orders for pressure are improved.

Copyright $@$ by SIAM. Unauthorized reproduction of this article is prohibited. 
5. Conclusions. In this paper, we have developed some numerical schemes for the FE approximation of the mixed formulation of the hydrostatic Stokes system or primitive equations of the ocean (without vertical integration), which allow us to use Stokes-stable FE elements and some computational techniques, like mesh adaptivity.

The $v$-stabilized scheme $(2.4)-(2.6)$ adds the consistent stabilizing term $(\nabla$. $\left.\left(\mathbf{u}_{h}, v_{h}\right), \partial_{z} \bar{v}_{h}\right)$ to the vertical momentum equation. In this way, the $H^{1}$-coercivity, which disappears in hydrostatic problems due to the lack of regularity of vertical velocity, is partially recovered, and thus we can prove (using a saddle-point argument) stability for some Stokes-stable FE, like Taylor-Hood $\mathcal{P}_{2}-\mathcal{P}_{1}$ or the minielement $\left(\mathcal{P}_{1}+\right.$ bubble $)-\mathcal{P}_{1}$ FE. In this case, optimal order is reached for $\mathcal{P}_{2}-\mathcal{P}_{1}$ but not for $\left(\mathcal{P}_{1}+\right.$ bubble $)-\mathcal{P}_{1}$. Former results are extended to the more realistic quasi-hydrostatic problem (adding the coercive term $\varepsilon^{2}\left(\nabla v_{h}, \nabla \bar{v}_{h}\right)$ ), and a symmetric reformulation of this scheme is also presented.

The $\partial_{z} v$-regularized scheme (3.11)-(3.13) adds the consistent term $\left(\partial_{z} p_{h}, \partial_{z} \bar{p}_{h}\right)$ to the $v$-stabilized problem. (This argument fails if it is added to the original non$v$-stabilized hydrostatic problem.) Using a generalized inf-sup condition and the Banach-Necas-Babuška's theorem, we prove well-posedness and error estimates with an additional bound of $\partial_{z} p$ in $L^{2}(\Omega)$. In this case, optimal order is reached with $\left(\mathcal{P}_{1}+\right.$ bubble $)-\mathcal{P}_{1}$ but not with $\mathcal{P}_{2}-\mathcal{P}_{1}$.

Numerical experiments support previous analysis and also offer us additional information. Specifically, we exploit these schemes, developing numerical tests for the simulation of hydrostatic flows using Stokes-stable FE with generic meshes. Domains include even cases with singularities, for instance, the cases without talus or with discontinuous bottom function, where the $v$-stabilized scheme, especially combined with the $\partial_{z} p$-regularized scheme, exhibits its efficiency. Our models made easy even the development of satisfactory $2 \mathrm{D}$ and $3 \mathrm{D}$ experiments in realistic domains.

\section{REFERENCES}

[1] P. AzÉRAD, Analyse et approximation du problème de Stokes dans un bassin peu profond, C. R. Acad. Sci. Paris Sér. I Math., 318 (1994), pp. 53-58.

[2] P. AzÉRAD, Analyse des Équations de Navier-Stokes en Bassin peu Profond et de l'Équation de Transport, Ph.D. thesis, Neuchatel, 1996.

[3] P. AzÉRAd AND F. Guillén, Mathematical justification of the hydrostatic approximation in the primitive equations of geophysical fluid dynamics, SIAM J. Math. Anal., 33 (2001), pp. 847-859.

[4] O. Besson And M.R. Laydi, Some estimates for the anisotropic Navier-Stokes equations and for the hydrostatic approximation, Math. Model. Numer. Anal, 26 (1992), pp. 855-865.

[5] S.C. Brenner and L.R. Scott, The Mathematical Theory of Finite Element Methods, Appl. Math. Texts, 3rd ed., Springer-Verlag, New York, 2008.

[6] F. Brezzi And M. Fortin, Mixed and Hybrid Finite Element Methods, Springer-Verlag, NewYork, 1991.

[7] C. CaO And E.S. Titi, Global well-posedness of the three-dimensional viscous primitive equations of large scale ocean and atmosphere dynamics, Ann. of Math., 166 (2007), pp. 245267.

[8] T. Chacón-Rebollo and F. Guillén-GonzÁlez, An intrinsic analysis of the hydrostatic approximation of Navier-Stokes equations, C. R. Acad. Sci. Paris, Sér. I Math, 330 (2000), pp. 841-846.

[9] T. Chacón-Rebollo and D. Rodríguez-Gómez, A numerical solver for the primitive equations of the ocean using term-by-term stabilization, Appl. Numer. Math., 55 (2005), pp. 131.

[10] B. Cushman-Roisin and J.M. Beckers, Introduction to Geophysical Fluid DynamicsPhysical and Numerical Aspects, Academic Press, New York, 2009.

[11] A. Ern and J.-L. Guermond, Theory and Practice of Finite Elements, Springer-Verlag, New York, 2004. 
[12] V. Girault and P.-A. Raviart, Finite Element Methods for Navier-Stokes Equations, Springer-Verlag, New York, 1986.

[13] F. Guillén-González, N. Masmoudi, and M.A. Rodríguez-Bellido, Anisotropic estimates and strong solutions of the primitive equations, Differential Integral Equations, 14 (2001), pp. 1381-1408.

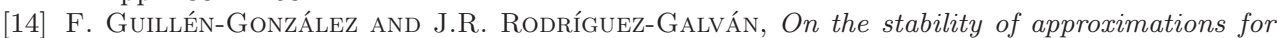
the Stokes problem using different finite element spaces for each component of the velocity, Appl. Numer. Math., to appear.

[15] F. Guillén-González and J.R. Rodríguez-Galván, Analysis of the hydrostatic Stokes problem and element approximation in unstructured meshes, Numer. Math., 130 (2015), pp. 225-256.

[16] F. Guillén-GonzÁlez And D. RodríGuez-Gómez, Bubble finite elements for the primitive equations of the ocean, Numer. Math., 101 (2005), pp. 689-728.

[17] Y. He, First order decoupled method of the primitive equations of the ocean I: Time discretization, J. Math. Anal. Appl., 412 (2014), pp. 895-921.

[18] Y. He, Second order decoupled implicit/explicit method of the primitive equations of the ocean I: Time discretization., Int. J. Numer. Anal. Model., 12 (2015), pp, 1-30.

[19] Y. HE AND J. Wu, Global H2-regularity results of the 3D primitive equations of the ocean, Int. J. Numer. Anal. Model., 11 (2014), pp. 452-477.

[20] F. Неснт, New development in freefem ++, J. Numer. Math., 20 (2012), pp. 251-265.

[21] M. Kimmritz And M. BRAack, Equal-order finite elements for the hydrostatic Stokes problem, Comput. Methods Appl. Math., 12 (2012), pp. 306-329.

[22] J.-L. Lions, R. Temam, And S. Wang, New formulations of the primitive equations of the atmosphere and applications, Nonlinearity, 5 (1992), pp. 237-288.

[23] J.-L. Lions, R. Temam, And S. Wang, On the equations of large scale ocean, Nonlinearity, 5 (1992), pp. 1007-1053.

[24] U.S.A. National Geophysical Data Center NOAA, 2-Minute Gridded Global Relief Data (etopo $2 v 2$ ), www.ngdc.noaa.gov $/ \mathrm{mgg} /$ global/etopo2.html.

[25] F. Ortegón Gallego, Regularization by monotone perturbations of the hydrostatic approximation of Navier-Stokes equations, Math. Models Methods Appl. Sci., 14 (2004), pp. 18191848.

[26] J. Pedlosky, Geophysical Fluid Dynamics, Springer-Verlag, New York, 1987.

[27] R. Tемам, Some mathematical aspects of geophysical fluid dynamic equations, Milan J. Math., 71 (2003), pp. 175-198.

[28] R. Temam and M. Ziane, Some mathematical problems in geophysical fluid dynamics, in Handbook of Mathematical Fluid Dynamics, Vol. 3, S. Friedlander and D. Serre, eds., Elsevier, New York, 2004, pp. 535-658.

[29] M. Ziane, Regularity results for Stokes type systems, Applicable Analysis, 58 (1995), pp. 263292.

Copyright (c) by SIAM. Unauthorized reproduction of this article is prohibited. 